\title{
AN END EFFECT ASSOCIATED WITH CYLINDRICAL LANGMUIR PROBES MOVING AT SATELLITE VELOCITIES
}

BY

RICHARD T. BETTINGER

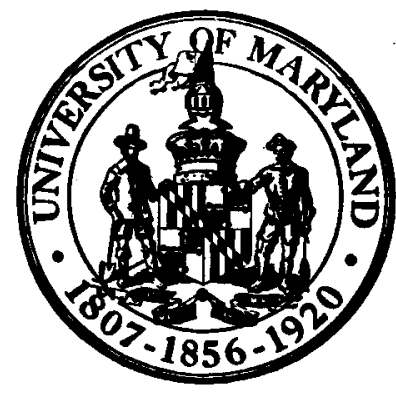

GPO PRICE

CFSTI PRICE(S)

Hard copy (HC)

3.00

Microfiche (MF)

.25

UNIVERSITY OF MARYLAND " 853 July 85

DEPARTMENT OF PHYSICS AND ASTRONOMY

COLLEGE PARK, MARYLAND

AUGUST 1966
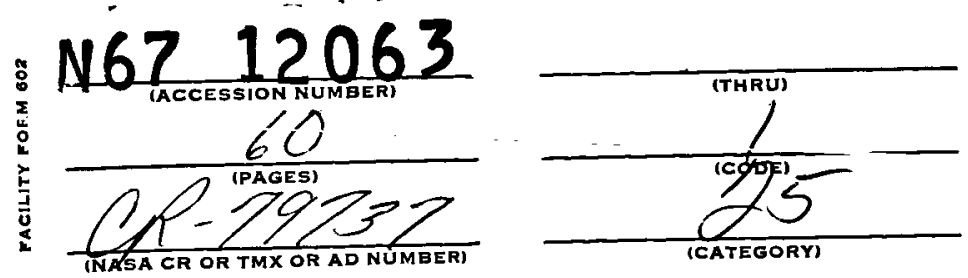


\section{AN END EFFECT ASSOCIATED WITH CYLINDRICAL LANGMUIR \\ PROBES MOVING AT SATELLITE VELOCITIES*}

by

Richard T. Bettinger

University of Maryland

Department of Physics and Astronomy

College Park, Maryland

August 1966

* Supported in part by the National Aeronautics and Space Administration Grant NGR-21-002-060. 
ABSTRACT

An anomaly in the volt-ampere characteristic of the cylindrical Langmuir probe carried aboard the Explorer XVII Satellite has been observed. This phenomena can be explained as an end effect which becomes important as the angle $(\theta)$ between the axis of the probe and the velocity vector of the satellite becomes small. An analytical expression for this end current as a function of $\theta$ is derived. This effect may be utilized to experimentally obtain information on the ion temperature and its mass distribution as well as the dimension of the sheath about the probe. It is also suggested that this effect might be utilized to accurately detect the velocity vector of the satellite. 


\section{INTRODUCTION}

Brace $^{1}$ has noted an anomaly in the ion current response of the cylindrical Langmuir probe carried aboard Explorer XVII. This consists of a relatively narrow peak with an amplitude which may be more than twice the normal response (see Fig. 1). This phenomenon appeared to occur on $1 y$ when the axis of the probe is oriented near the velocity vector of the satellite.

This current anomaly results from the finite length of the detector, (i.e. an end effect). When considering an infinite probe whose axis is oriented parallel to the velocity vector of the satellite (Fig. 2 with $\theta=0$ ), one obtains a current equivalent to that of a probe at rest. The open end of a probe of finite length, however, represents a hole in the dike which gives rise to a current contribution which is related to the satellite velocity. As an extreme illustration, let us consider the case of a cool plasma. The current through the sides of a cylindrical probe, whose radius is small compared to that of the sheath, is given by Mott-Smith and Langmuir ${ }^{2}$ as

$$
i_{t}=r l_{p} n_{i} e\left[\frac{8 k T}{m_{i}}(1+\Phi)\right]^{\frac{1}{2}},
$$

where $r$ is the probe radius; $\ell_{p}$ is the probe length; $n_{i}$ is the ambient ion density; $e$ is the electronic charge; $k$ is the Boltzmann constant; $\mathrm{T}$ is the temperature; $\mathrm{m}_{i}$ is the ion mass and $\Phi$ is the nondimensional probe potential given by

$$
\Phi=\frac{\mathrm{eV}_{\mathrm{p}}}{\mathrm{kT}},
$$




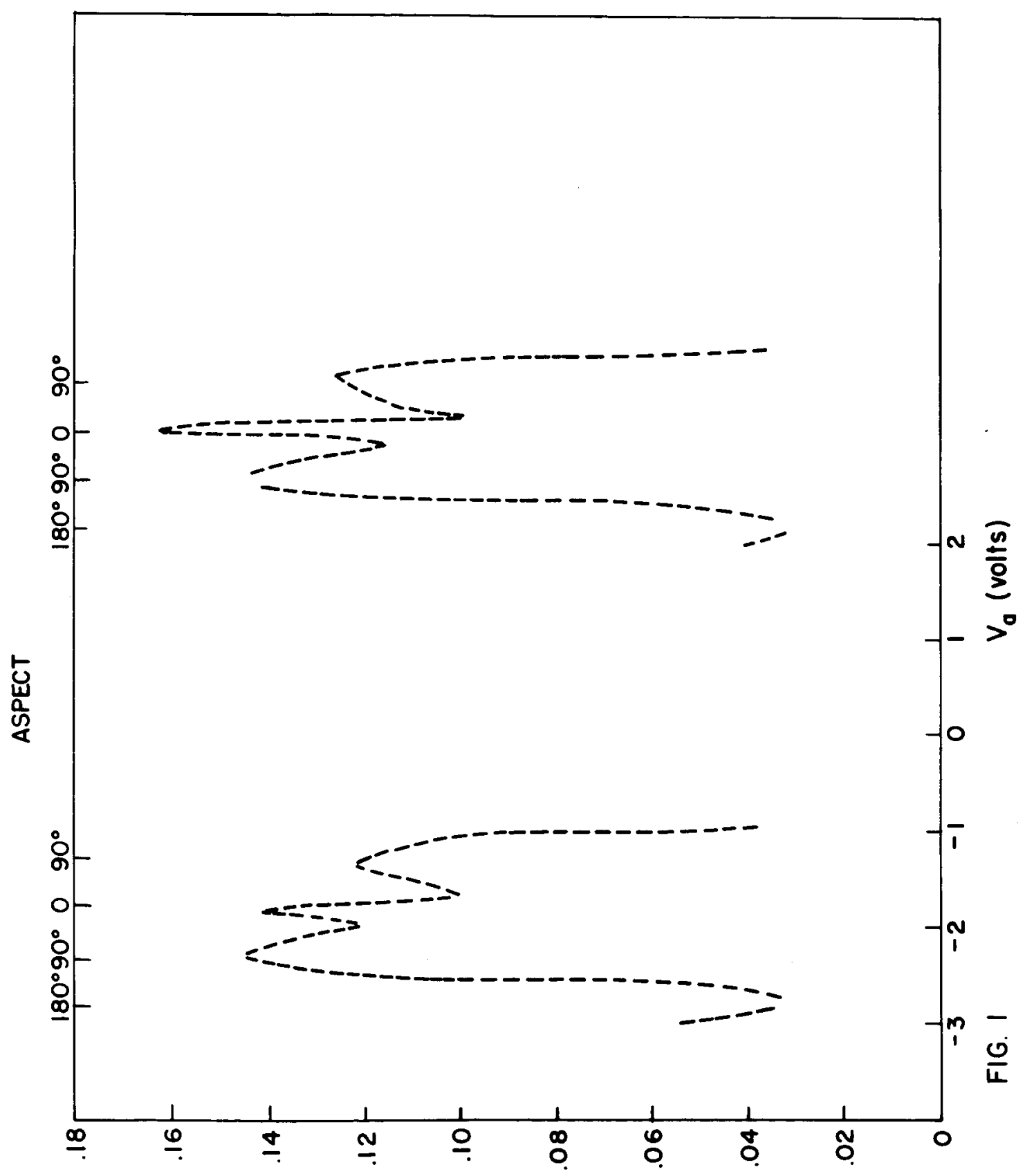




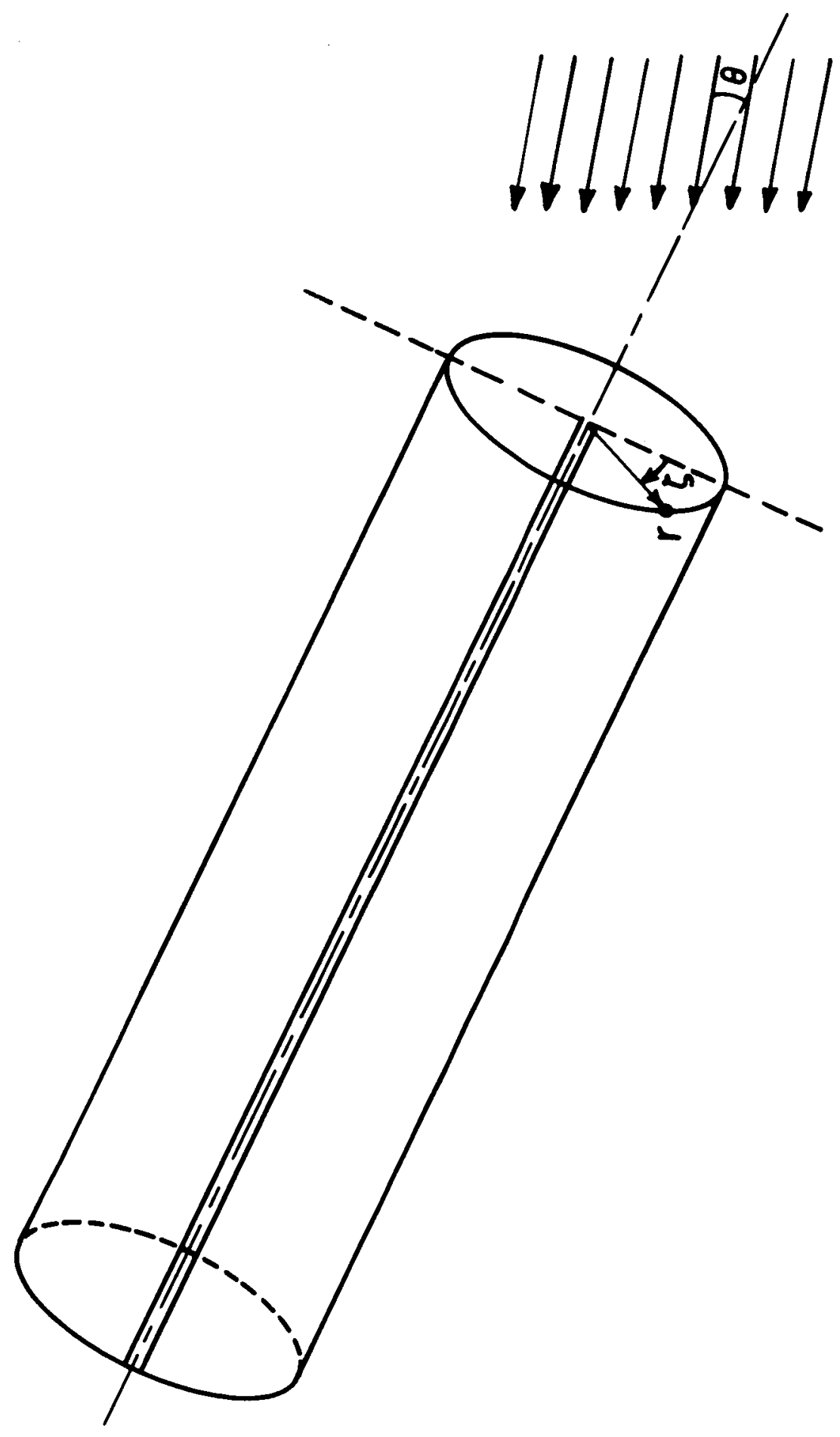


where $V_{p}$ is the probe to plasma potential. The current through the end of the sheath is given by

$$
i_{e}=2 \pi a^{2} n_{i} u,
$$

where $a$ is the sheath radius and $u$ is the satellite velocity. It is clear that by suitable choice of the parameters $i_{e}$ may be made arbitrarily large as compared to $i_{t}$; in particular, if the temperature is not too large, $r$ can be made arbitrarily small without affecting $i_{e}$. The latter is a "mechanical property" of the system and, as we shall see, gives rise to some interesting behavior.

This paper concerns itself with this anomalous end current and examines the influence of various factors on this phenomenon. 
COLD PLASMA

We begin by examining the case where the plasma is cold (i.e., $\left.T_{i}=T_{e}=0\right)$. We choose a set of cylindrical coordinates $(r, \zeta, z)$ such that the $z$ axis lies along the axis of the probe and with the origin coinciding with the tip of the probe. We will assume that all ions incident on the plane $(z=0)$ within the radius $(r=a)$ experience the same change in potential in the $(r, \zeta)$ plane as if they were accreted through the side of the sheath. (This point will be discussed in detail in the following section.) In the case of an earth satellite the vehicle velocity $u$ is, in general, large compared with the ion velocities and small as compared with that of the electrons. We assume that the probe potential is negative and large enough so that electron currents can be neglected. In this frame of reference, we have incident on the sheath of our probe, an omnidirectional beam of monoenergetic ions. Let us restrict our attention to their motion in the $(r, \zeta)$ plane. If the radial distribution of potential may be represented as a power law

$$
\phi=\mathrm{Kr}^{-\mathrm{m}},
$$

where $\mathrm{m}$ is less than two then the closest appraoch of any given ion to the axis $(r=0)$ is determined by its angular momentum. From the conservation of angular momentum $L$, we may write

$$
\mathrm{L}=\mathrm{r}^{2} \dot{\zeta}=\text { constant }
$$


where the dot refers to the time derivative.

The conservation of energy leads to

$$
\frac{1}{2} m_{i}\left(\dot{r}^{2}+r^{2} \dot{\zeta}^{2}+\dot{z}^{2}\right)=\frac{1}{2} m_{i}\left(\dot{r}_{1}^{2}+r_{1}^{2} \zeta_{1}^{2}+\dot{z}_{1}^{2}\right)-\phi \quad,
$$

where the subscript refers to points beyond the plane $(z=0)$. The velocity components may be written as

$$
\begin{aligned}
\dot{r} & =u \sin \theta \cos \zeta \\
r \dot{r} & =u \sin \theta \sin \zeta \\
\dot{z} & =u \cos \theta .
\end{aligned}
$$

Our assumption regarding the potential permits us to write

$$
\dot{z}=\dot{z}_{1},
$$

hence, we need only consider the two dimensional problem if we further assume the probe is sufficiently long to guarentee collection. Combining equations (5), (6) and (7), we obtain

$$
\dot{\mathrm{r}}_{1}^{2}+\frac{\mathrm{L}^{2}}{\mathrm{r}_{1}^{2}}-\frac{2 \phi}{\mathrm{m}_{i}}=\mu^{2} \sin ^{2} \theta
$$

The radius of closest approach is obtained by setting $\dot{\mathrm{r}}_{1}=0$, hence,

$$
r_{c}=|L|\left(\mu^{2} \sin ^{2} \theta+\frac{2 \phi}{m_{i}}\right)^{-\frac{1}{2}} .
$$

This clearly demonstrates the one to one dependence of the radius of closest approach and the angular momentum.

The condition for collection by the probe, assuming no reflectance, is

$$
\mathrm{r}_{\mathrm{c}} \leq \mathrm{r}_{\mathrm{p}}
$$


where $r_{p}$ is the probe radius. The equivalent condition in terms of the angular momemtum is

$$
L^{2} \leq r_{p}^{2}\left(u^{2} \sin ^{2} \theta+\frac{2 \phi}{m_{i}}\right)
$$

therefore, substituting (7b) into (12) we obtain a limiting relation between $r$ and $\zeta$ such that

$$
\left(r / r_{p}\right)^{2} \leq \csc ^{2} \zeta\left(1+\frac{\phi}{U \sin ^{2} \theta}\right)
$$

where

$$
\mathrm{U}=\frac{1}{2} \mathrm{~m}_{\mathbf{i}} \mathrm{u}^{2}
$$

For sufficiently small values of $\zeta$, particles at all radii from zero to a (the sheath radius), will be collected. There exists an angle $\zeta_{0}$, however, beyond which particles begin to be lost. This angle is given by

$$
\sin \zeta_{0}=\left(\frac{r_{p}}{a}\right)\left(1+\frac{\phi}{U \sin ^{2} \theta}\right)^{\frac{1}{2}} \quad 0 \leq \zeta_{0} \leq \frac{\pi}{2} .
$$

The total current to the probe, through the end of the sheath is, therefore,

$$
i_{e}=n_{i} e \mu \cos \theta\left\{\int_{0}^{2 \pi} \int_{0}^{r_{p}} \operatorname{rdrd} \zeta+4 \int_{0}^{\zeta_{0}} \int_{r_{p}}^{a} \operatorname{rdrd} \zeta+4 \int_{\zeta_{0}}^{\zeta_{l}} \int_{r_{p}}^{r_{e}} r d r d \zeta\right\} .
$$

The first term is merely the physical area of the probe. The second term includes the region where the critical radius is greater than the sheath dimensions. The final term takes into account the region of diminishing area and includes an upper limit on the angle, $\zeta_{1}$ where

$$
0 \leq \zeta_{1} \leq \frac{\pi}{2}
$$


which is defined as the point at which $r_{\ell}=r_{p}$. That is

$$
\sin \zeta_{1}=\left(1+\frac{\phi}{U \sin ^{2} \theta}\right)^{\frac{1}{2}},
$$

It is evident that equation (17) is never satisfied except as a limiting condition, hence, we may replace $\zeta_{1}$ with $\pi / 2$ in (16). After integration, we obtain

$$
i_{e}=n_{i} e \mu \cos \theta a^{2}\left(2 \zeta_{0}+\sin 2 \zeta_{0}\right)
$$

The variation of the normalized form factor $\left[\frac{1}{\pi}\left(2 \zeta_{0}+\sin 2 \zeta_{0}\right)\right]=G$ with $\zeta_{0}$ is shown in Fig. 3. We define a half width in terms of the angle $\zeta$ at which $G=0.5$. The dependence of this function on the controlling parameters may be seen in Fig. 4 and 5 . You will note that for typical satellite conditions (i.e., the Explorer XVII electrostatic probe) $\frac{r_{p}}{a}=0.028$ and $\phi / U=0.5$, the half width is less than $3^{\circ}$. It is also of some interest to note that the width varies neariy linearly with probe to sheath radii ratio in this regime.

While the "cold plasma" case is very interesting, it has virtually no application in the ionosphere. The condition of applicability can be defined as

$$
\mu \sin \theta_{h} \gg\left(\frac{2 k T_{i}}{m_{i}}\right)^{\frac{1}{2}},
$$

where $\theta_{h}$ is the half width. The two sides of (19) are generally of the same order of magnitude so that we must include the effects of plasma temperature in our analysis. 
9

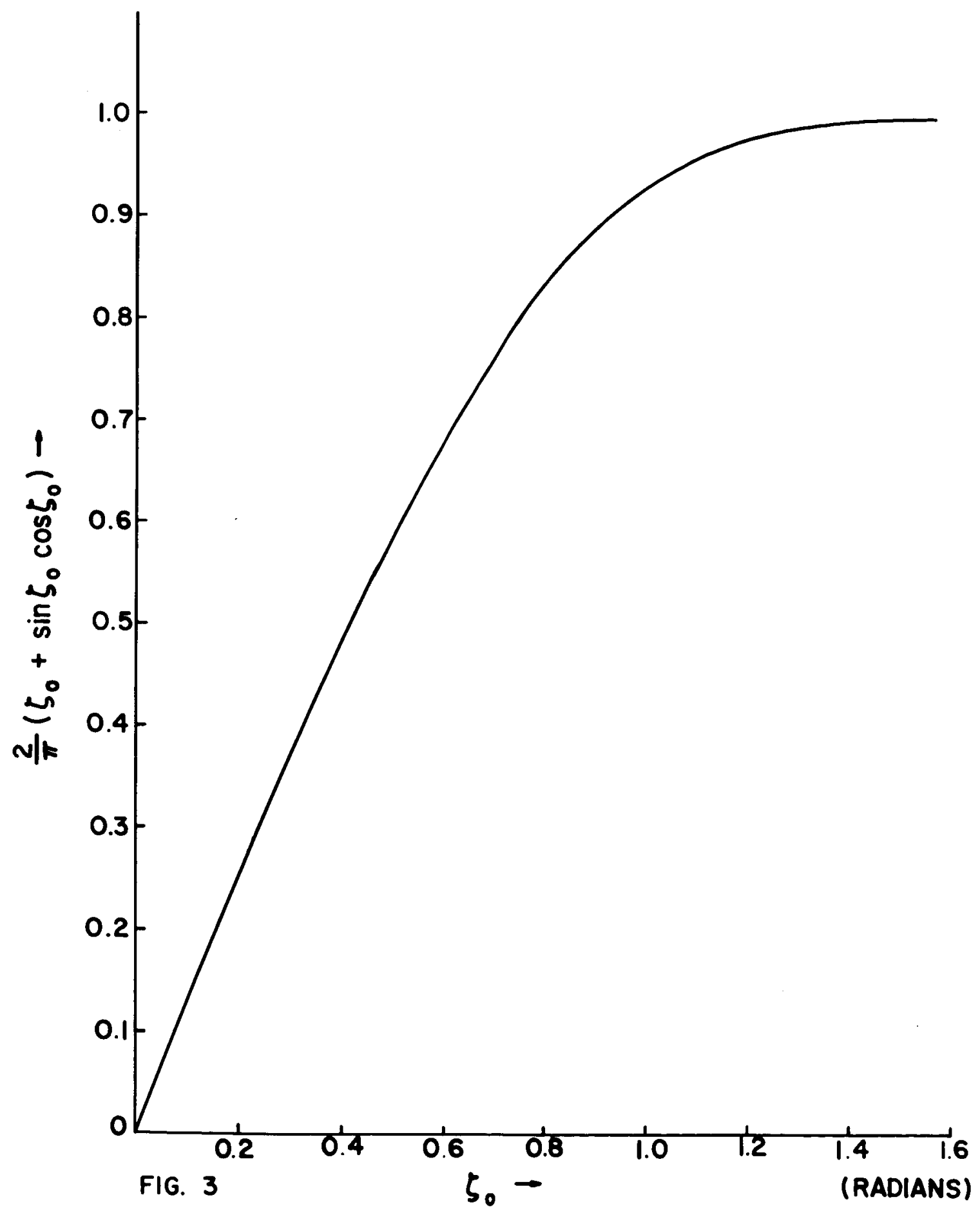




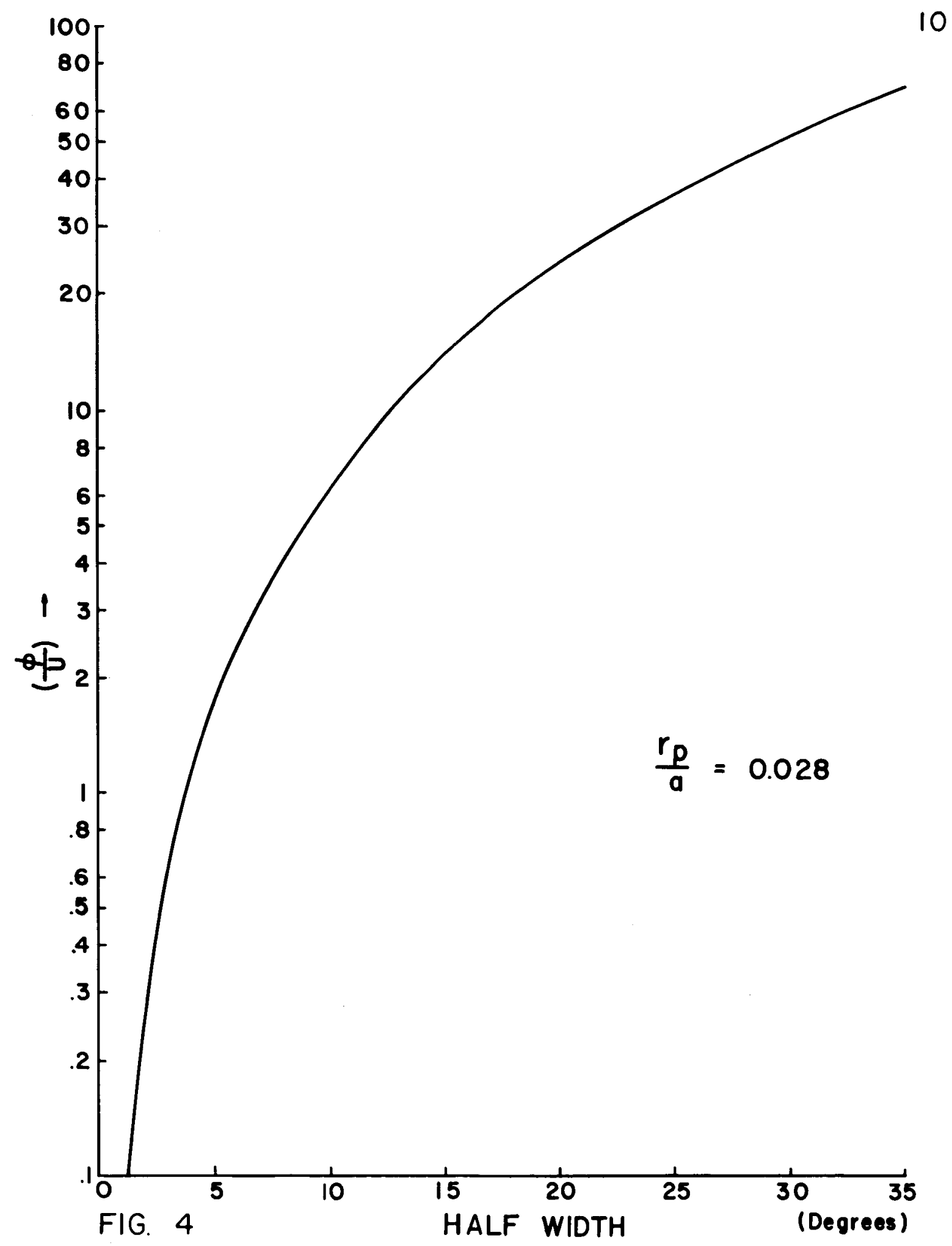




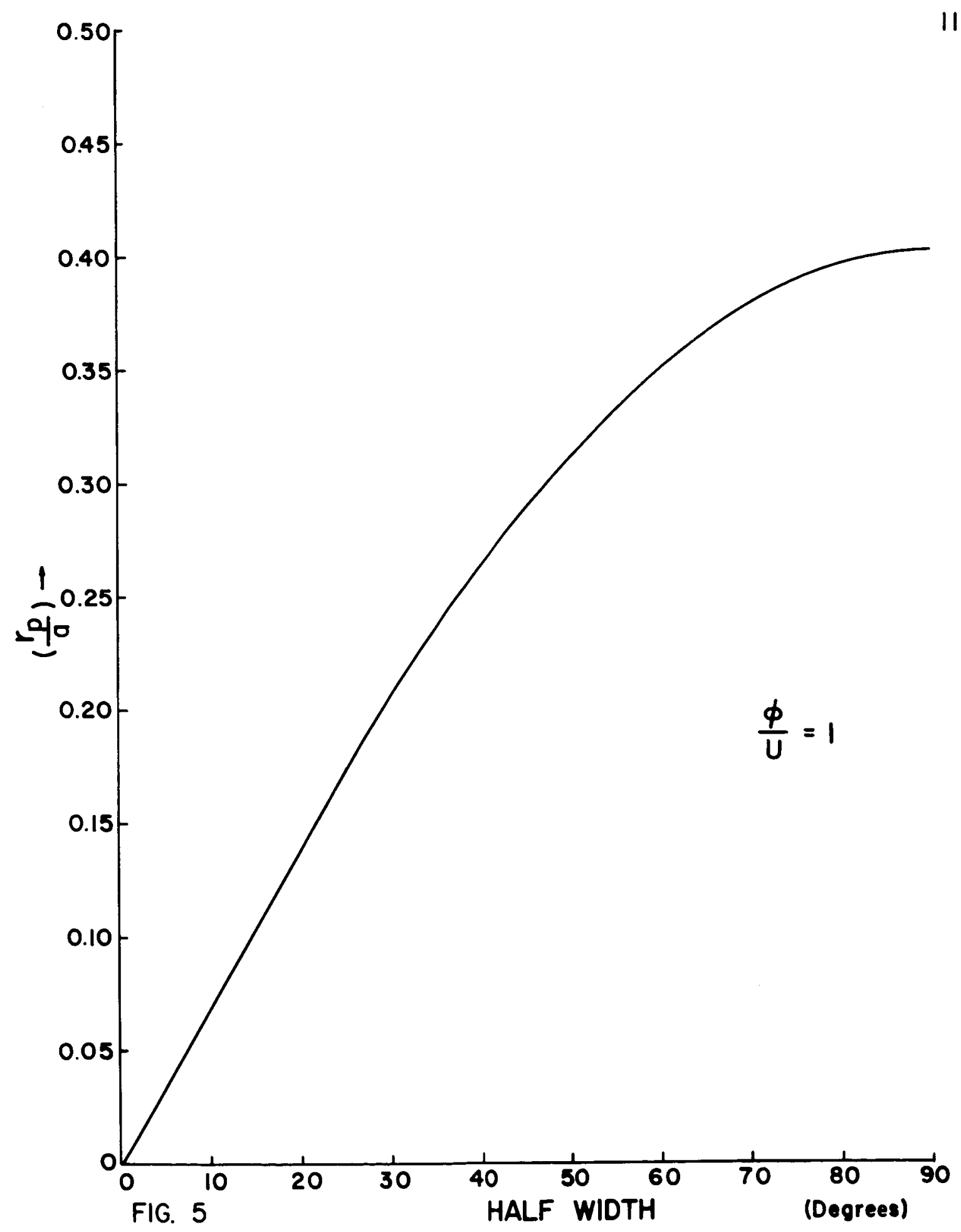


SHEATH CONSIDERATIONS

Before proceeding further it seems appropriate to consider some properties of the sheath as they relate to this problem. As was previously indicated, the factor which limits the collection of ions incident on the sheath is conservation of angular momemtum. If we consider motion in the $r-\zeta$ plane, this requires

$$
E_{\zeta}=\frac{L^{2}}{r^{2}} \frac{m}{2},
$$

where $E_{\zeta}$ is the component of the kinetic energy in the $\zeta$ direction. Thus, the rotational energy increases inversely as the square of the radius and this must be supplied from the change in potential energy or, if this is insufficient, by a decrease in the radial component of energy. With the latter condition, a particle eventually reaches a point at which the radial velocity vanishes and it is reflected.

It is evident that reflection can not occur in any region where the potential change with radius is faster than

$$
\phi=\frac{\mathrm{K}}{\mathrm{r}^{2}} \quad
$$

and where

$$
\mathrm{K}^{2} \geq \mathrm{L}^{2}
$$

If these conditions are satisfied between radii $r_{1}$ and $r_{2}\left(r_{2}\right.$ greater than $r_{1}$ ), then any accelerated particle incident on $r_{2}$ must eventually pass to surface $r_{1}$. If $r_{1}$ is coincidental with the surface of the probe, then the effective diameter of the probe becomes $r_{2}$. It must, however, be emphasized that the condition for reflection is completely specified by Eq. (6) and a detailed knowledge of the potential distribution 
between the sheath and the point of interest is not necessary. Rather, we need only know the potential at the point of interest relative to that at the boundary. There are some restrictions on the potential distribution, however, for our present purposes a sufficient condition to validate these conclusions is that the potential be a monotomic function.

The potential for the unshielded probe falls of $f$ as $\log r$ in the case of cylinder and is $\frac{1}{r}$ for a sphere. The effect of shielding is to cause the potential to change more rapidly and to converge to zero within the finite distance from the probe.

We would like to estimate the conditions under which "pericritical orbits"* are of importance. We assume that the potential may be written as a simple power law of the form

$$
\phi=\mathrm{Kr}^{-\mathrm{m}},
$$

where $\mathrm{K}$ and $\mathrm{m}$ are constants. The gradient of the field is therefore

$$
-|\overline{\mathrm{E}}|=\Delta \phi=\frac{\delta \phi}{\delta \mathrm{r}}=-\mathrm{mKr} \mathrm{r}^{-\mathrm{m}-1}
$$

Applying Gausses theorem

$$
\int_{S} \bar{E} \cdot d \bar{s}=\int_{V} \frac{\rho}{e_{o}} d V,
$$

\footnotetext{
* This terminology was introduced by $\mathrm{Opik}^{4}$ to distinguish those particle orbits which, because of the nature of the field and the relative energy of the particle, must spiral into the collector.
} 
we obtain after substitution of (23) for $|\bar{E}|$ and integrating

$$
2 \pi m K r-m=\frac{Q}{e_{o}},
$$

where $Q$ is the charge per unit length contained within the surface given by radius $r$. Substituting (22) into (25) and solving for $\mathrm{m}$, we obtain

$$
\mathrm{m}=\frac{\mathrm{Q}}{2 \pi e_{\mathrm{o}} \phi}
$$

From (24) it is evident that the distribution of charge within the volume is unimportant so that we can take the charge to be uniformly distributed over the surface $r$. This suggests a capacitor of concentric cylinders whose capacity per unit length is of the form

$$
C=\frac{2 \pi e_{0}}{\ell_{\mathrm{n}}\left(\mathbf{r}_{2} / \mathbf{r}_{1}\right)}
$$

where $r_{1}$ and $r_{2}$ are the diameters of the cylinders and $r_{1}<r_{2}$. Substituting (27) into (26) noting $C=\frac{Q}{\phi}$

$$
\frac{1}{m}=l_{n}\left(\frac{r_{2}}{r_{1}}\right)
$$

The analogy of the sheath in terms of a cylindrical capacitor is obviously incomplete since the charge outside $r_{1}$ is not uniformly distributed on $r_{2}$; however, we take $r_{2}$ to be related to the effective sheath radius $a$, anticipating that $\left(a>r_{2}\right)$.

We find that this approach predicts a "pericritical" potential field $(m>2)$ only for large values of the ratio $\left(\frac{r_{1}}{r_{2}}\right)$ specifically

$$
r_{1} \geq 0.66 r_{2}
$$


Thus the potential distribution about a small probe $\left(r_{p}<a\right)$ never satisfies the pericritical condition except near the sheath edge. Therefore, we need not consider this problem in the present analysis, however, the apparent tendency to form a pericritical shell near the sheath "boundary" supplies a physical basis for the concept of a finite sheath edge as applied to collectors.

Since the charges of interest in the present context enter the sheath through the open "end", we need to know something about the potential distribution in this area. We restrict our consideration to probes with radial dimensions small compared with those of the sheath. Hence, the exact shape of the electrode tip is unimportant. Calculation of the appropriate distribution is a complex problem involving solution of Poisson's equation by numerical techniques. This problem has been treated by a number of investigators $5,6,7$ using various approximations. Probably the most general approach is that of Walker 8 where the main assumption which limits the applicability of his work is that all charges are reflected at the surface of the collector. This introduces errors, primarily at the sheath edge, but these are not important except for large bodies. The vast majority of the charge carriers which enter the sheath of a small collector of the type which we are presently considering are reflected by the angular momenta considerations previously discussed and, in general, only a very small fraction reach the collector. In the case of the end effect under consideration, we do collect an abnormal percentage of ions incident on the end of the sheath, but the bulk of these are collected at large distances from the probe tip. The sheath, remote from the tip, is largely constructed of charges incident through its sides so 
that again the charges collected are numerically much less than the total number which enters the entire sheath. If follows, therefore, that we can employ the Walker technique to this problem with some degree of confidence.

Unfortunately, Walker calculations employing cylindrical geometry have not as yet been completed, but it seems apparent that the sheath about the probe tip will not differ significantly from that of a small sphere of the same radius. We also expect that the relatively high probe velocity with respect to the ion thermal velocities will not have a major effect on the sheath structure over the forward tip of the probe. Fig. 6 is a result from Walker 8 , where the density distribution of ions is plotted for a small body moving at typical satellite velocities. The corresponding potential field is shown in Fig. 7.

The odd shape of the body results from the calculation technique employed by Walker. He assumes an arbitrary, small potential on a surface at a large distance from the origin and then calculates inward. The body shape is then any resulting equipotential surface which can be arbitrarily selected. Figure 8 compares the potential distributions calculated for a moving body and one at rest. The latter case assumes a sphere with a radius of one Debye length, i.e., $\rho=1$ where the Debye length (h) is given by

$$
h=\left(\frac{k T}{4 \pi n e^{2}}\right)^{\frac{1}{2}} \text {. }
$$

While the geometries are only approximately equivalent, it is apparent that the results do not differ greatly. This follows from the fact that the ion density distribution over the front of the body is not greatly affected by its presence and the electron thermal velocities are large 


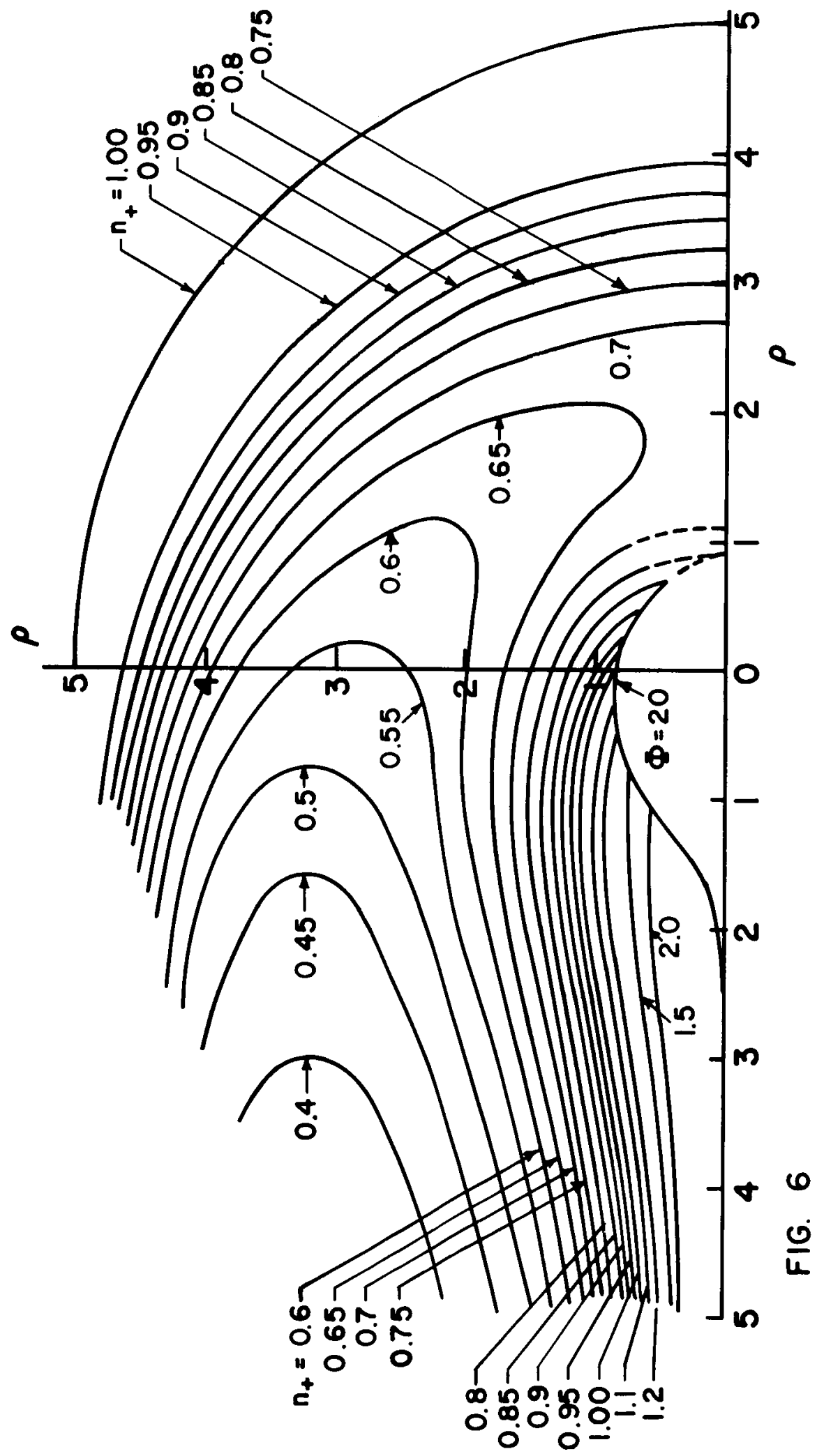




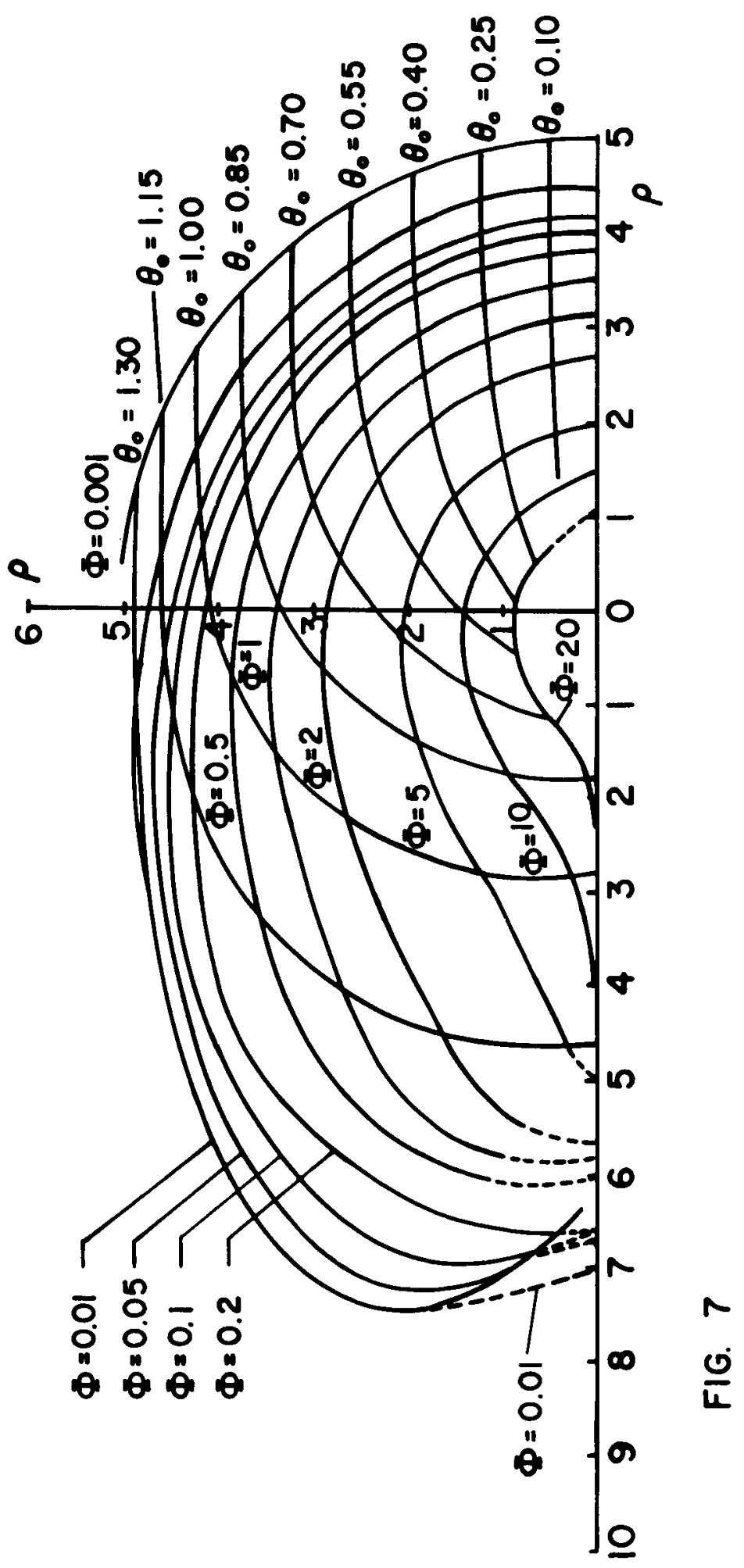




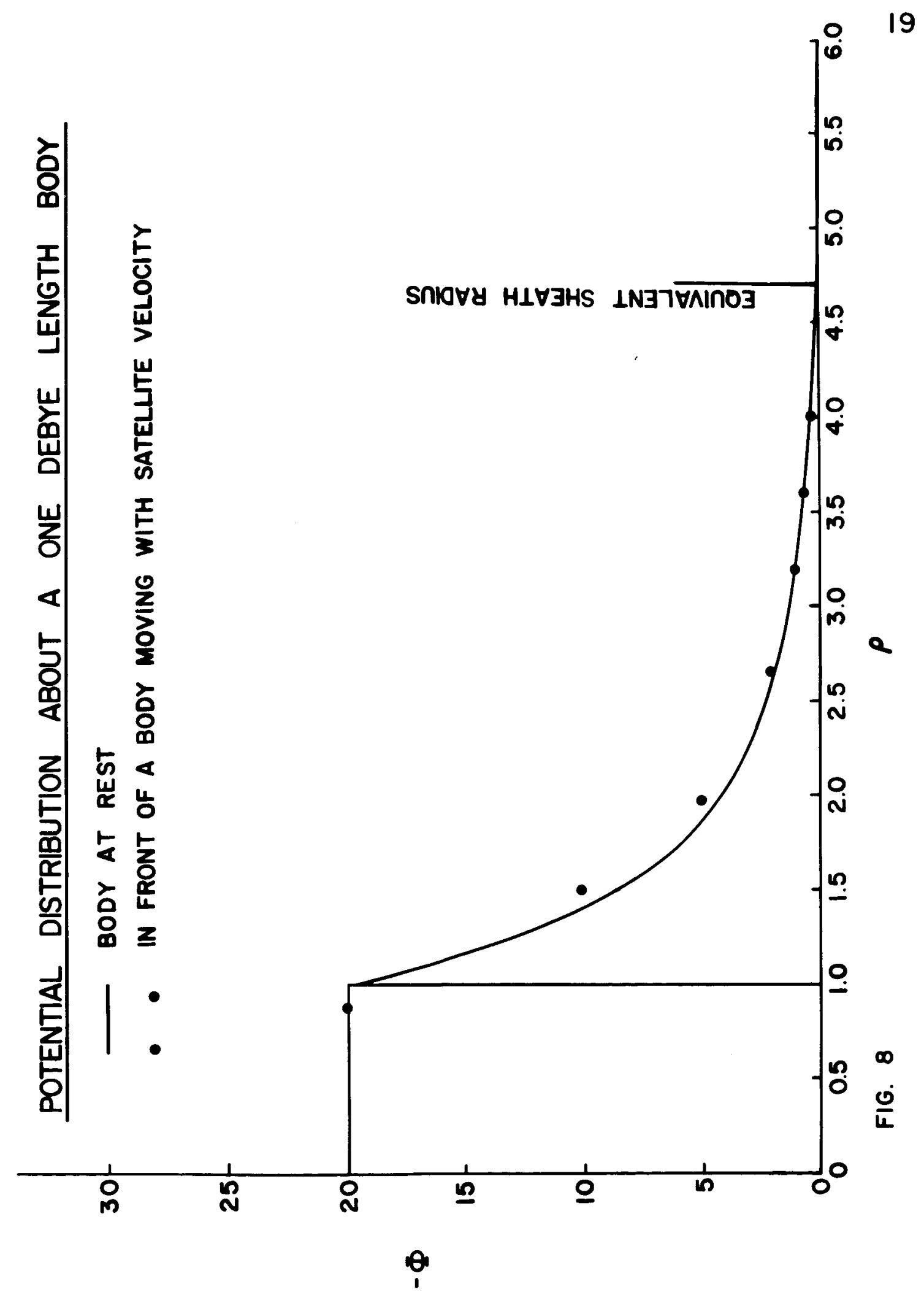


compared with the satellite velocity. The sheath is constructed, therefore, in a field of ion charges of roughly uniform density (within a factor of 2) by pushing out the electrons until the sheath contains a sufficient excess of positive ions to balance the charge carried by the body. Hence, although the sheath is made up of positive charges, it is modulated by rearranging the electrons. This is particularly true at satellite velocities where the ion energies are of the order of the probe potential ( $V_{i}$ typically 5.5 Volts) making the ions "stiff". This is not the case for very large bodies, however, it does apply to spheres with radii up to several Debye lengths. For instance, Fig. 9 (from Walker ${ }^{8}$ ) plots normalized ion density versus $\rho_{0}-\rho$ where $\rho_{0}$ is the initial point at some distance from the body and is the nondimensional radius (in Debye lengths). It is evident that the density remains relatively close to unity, even for relatively large bodies, until the convergence of space causes it to rise rapidly. We conclude that we can, with reasonable accuracy, apply the Walker calculations for very small spheres at rest. If we take the following parameters as "typical", we can examine a specific situtation.

$$
\begin{aligned}
& \mathrm{v}_{\mathrm{p}}=3.0 \text { Volts } \\
& \mathrm{n}_{e}=10^{5} \mathrm{el} / \mathrm{cc} \\
& \left.\mathrm{m}_{\mathrm{i}}=16 \mathrm{~m}_{\mathrm{p}} \text { (i.e., } 0^{+}\right) \\
& \mathrm{T}_{\mathrm{e}}=2500^{\circ} \mathrm{K} \\
& \mathrm{r}_{\mathrm{p}}=0.026 \mathrm{~cm}
\end{aligned}
$$

This leads to a Debye length of slightly more than $1 \mathrm{~cm}$ and a nondimensional probe radius $(\rho)$ of 0.026 . The nondimensional probe potential 
21

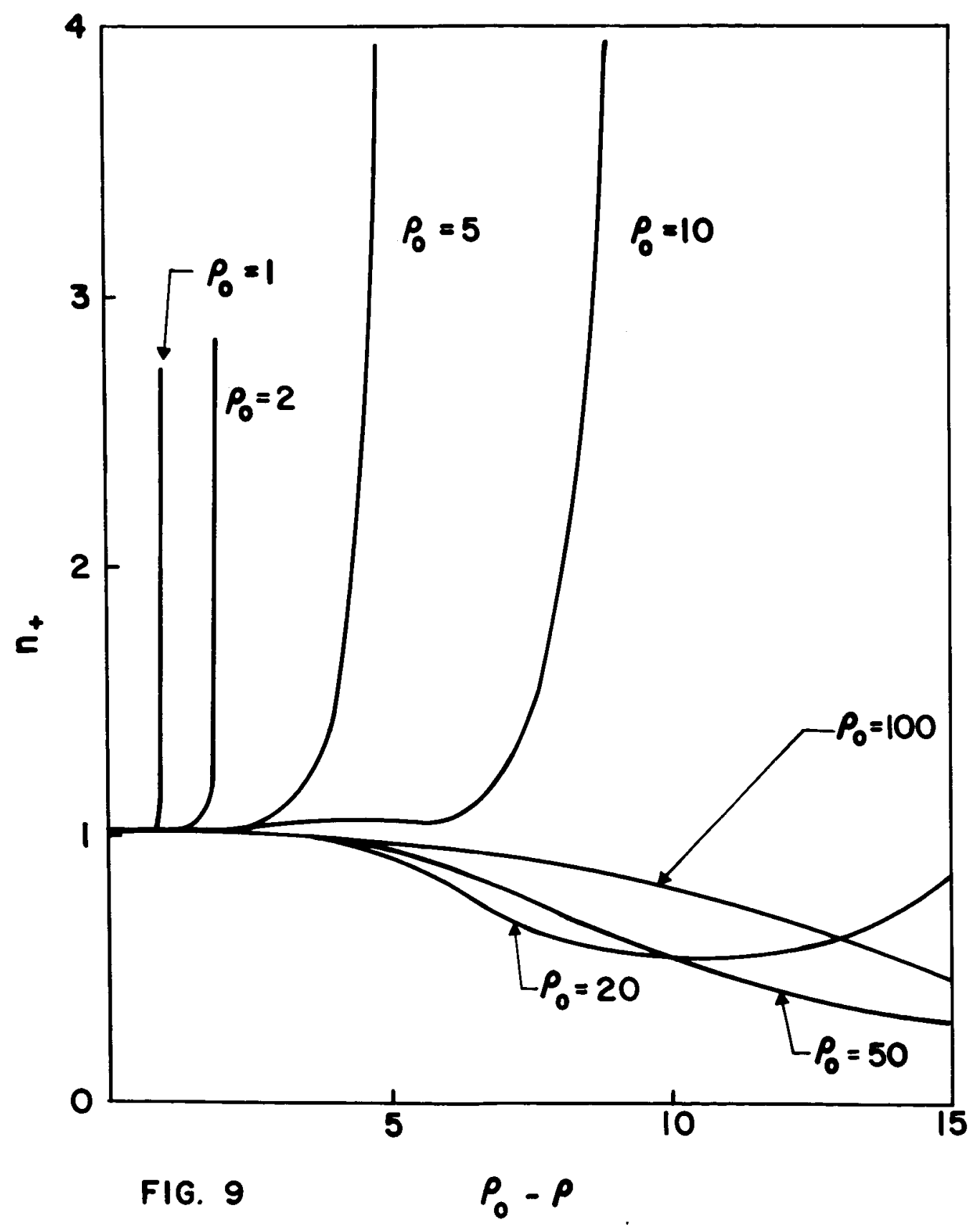


$\left(\phi_{\mathrm{p}}\right)$ is approximately 10 . Figure 10 displays the variation of potential with radius based on Walker's work along with a curve for which

$$
\phi=\mathrm{K} / \rho \quad,
$$

which represents the limiting case without shielding. Any calculation, which takes into account the effects of shielding must fall below this reference curve. As one might expect, the major difference between these curves occurs at large distances. We have indicated the effective sheath radius based on the equations developed by Bettinger and Walker ${ }^{10}$ where, in the case of the sphere, the sheath radius $\left(\rho_{\mathrm{a}}\right)$ in Debye lengths is given as

$$
\rho_{\mathrm{a}}=\rho_{\mathrm{p}}+0.83 \rho^{\frac{1}{3}} \psi^{\frac{1}{2}} \quad \text {. }
$$

The important point with regard to the present problem is the fact that the potential is large only very near to the probe. Particles entering the sheath through its end undergo an acceleration along the $z$ axis (see Fig. 2), so that depending upon the radius of initial penetration of sheath boundary, a particle will gain an energy $\phi^{\prime}$ in the $r-\zeta$ plane where

$$
0 \leq \phi^{\prime} \leq \phi_{p}
$$

Thus Eq. (15) should be replaced by

$$
\sin \zeta_{0}=\left(\frac{\rho_{p}}{\rho a}\right)\left(1+\frac{\phi^{\prime}}{U^{\prime} \sin ^{2} \theta}\right)^{\frac{1}{2}},
$$

where

$$
U^{\prime}=\phi_{p}-\phi^{\prime}+\frac{1}{2} m \mu^{2}
$$


23

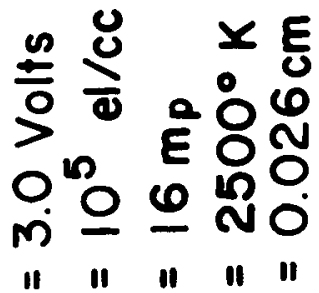

>른
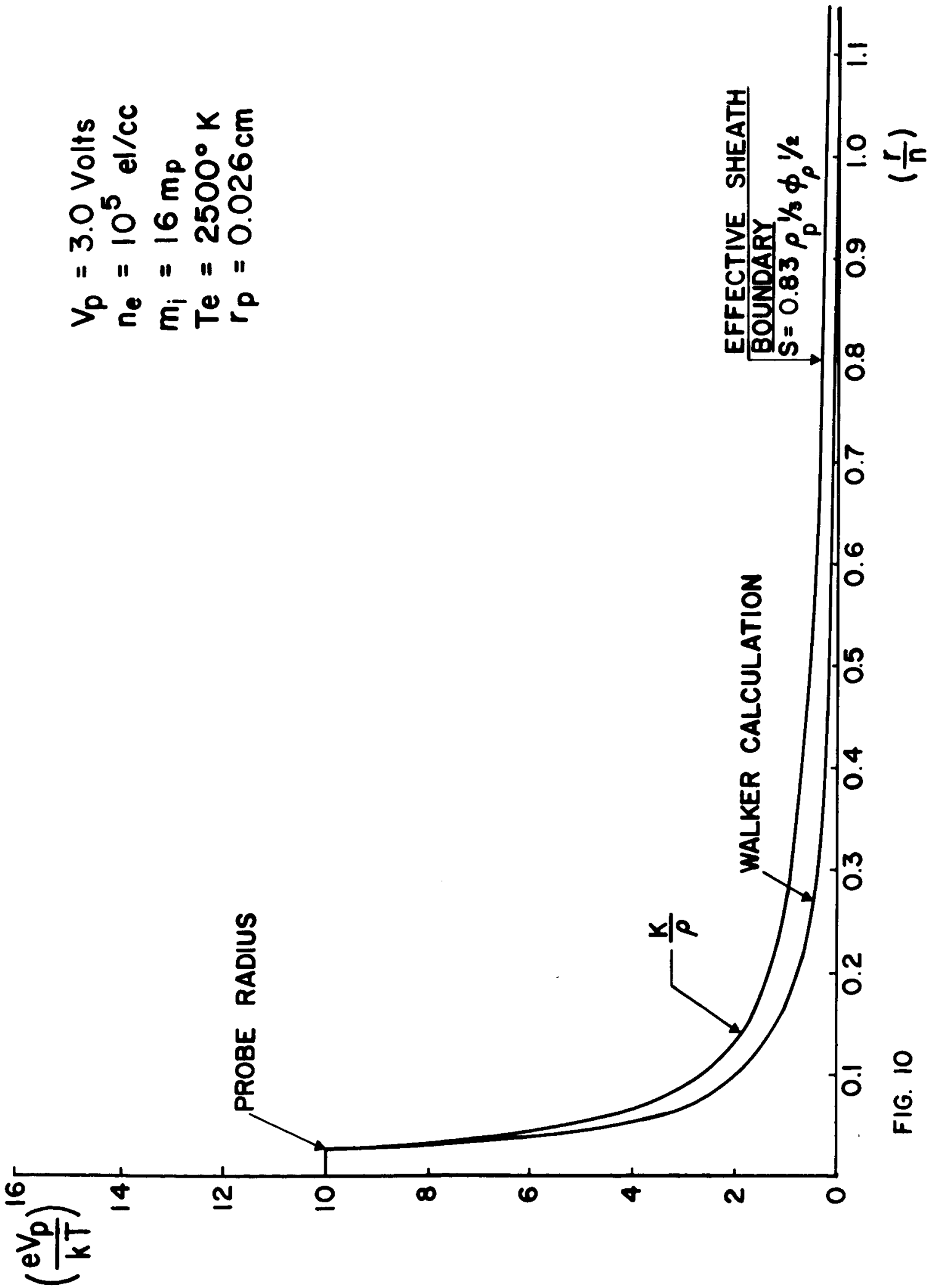
Given the potential function $[\phi=f(\rho)]$ we may readily obtain the reduced potential in terms of the impact radius $\rho_{i}$. In order to estimate the importance of this correction we calculate the average value of the reduced potential $(\bar{\phi})$. We can represent potential, as calculated by Walker, to good accuracy (See Fig. 10) with the function

$$
\phi(\rho)=\frac{0.0418}{\rho \frac{3}{2}}
$$

The reduced potential is simply the probe potential less the initial value of the parameter in the $r-\zeta$ plane. If we ignore the small radial displacement of particles as they pass through the sheath end, we may write

$$
\bar{\phi}^{\prime}=\phi_{p}-\frac{1}{A} \int_{0}^{2 \pi} \int_{\rho_{p}}^{\rho_{a}} \phi\left(\rho_{i}\right) \rho_{i} d \rho_{i} d w,
$$

where $A$ is the area of integration

$$
A=\pi\left(\rho_{a}^{2}-\rho_{p}^{2}\right) \simeq \pi \rho_{a}^{2},
$$

which is over a disc in the $r-\zeta$ plane at $z=0$ with the nondimensional impact parameter as the integration variable. This yields the result

$$
\begin{aligned}
\bar{\phi}^{\prime} & =\phi_{\mathrm{p}}-4 \phi_{\mathrm{a}}\left\{1-\left(\frac{\rho_{\mathrm{p}}}{\rho}\right)^{\frac{1}{2}}\right\} \\
& \simeq \phi_{\mathrm{p}}\left\{1-4\left(\frac{\rho_{\mathrm{p}}}{\rho_{\mathrm{a}}}\right)^{\frac{3}{2}}+\left(\frac{\rho_{\mathrm{p}}}{\rho_{\mathrm{a}}}\right)^{2}\right\}, .
\end{aligned}
$$

Using the "typical" values previously calculated $\left(\rho_{\mathrm{p}} / \rho_{\mathrm{a}}=0.026\right)$, the correction term is less than $2 \%$. Since the sheath radius (a) proceeds approximately inversely as the root of the charge density $\left(n_{e}\right)$, we would 
except that we can ignore the second and third terms of (38) for probes of this scale throughout the ionosphere.

It should be noted that the situation is not quite as favorable as is indicated above since charges with small impact parameters have a higher probability of collection. 


\section{WARM PLASMA}

We now turn our attention to the case of a warm plasma, that is, we assume that each plasma constituent possesses a Maxwellian distribution of velocities characterized by a temperature (T) and a distribution function

$$
\mathrm{d}^{3} \mathrm{~N}=\mathrm{N}_{\mathrm{o}}\left(\frac{\mathrm{m}}{2 \pi k \mathrm{~T}}\right)^{\frac{3}{2}} \quad \exp \left(\frac{\mathrm{E}}{\mathrm{kT}}\right) \mathrm{r}^{\prime} \mathrm{d} \dot{\mathrm{r}}^{\prime} \mathrm{d} \dot{\bar{\xi}}^{\prime} \mathrm{d} \dot{\mathrm{z}}^{\prime},
$$

where the dot refers to the time derivative and where the energy (E) is given by

$$
E=\frac{m}{2}\left\{\left(\dot{r}^{\prime}\right)^{2}+\left(r^{\prime} \dot{\zeta}\right)^{2}+\left(\dot{z}^{\prime}\right)^{2}\right\}
$$

The coordinate system $\left(r^{\prime}, \zeta^{\prime}, z^{\prime}\right)$ is a fixed frame in which there is no net plasma motion. Let us consider a frame $(r, \zeta, z)$ (see figure 2) fixed with respect to the probe and moving with respect to our initial frame such that the velocity components are related by

$$
\begin{aligned}
\dot{r} & =\dot{r}^{\prime}+\mu \sin \theta \cos \zeta \\
\dot{r} \dot{\zeta} & =r^{\prime} \dot{\zeta}^{\prime}+\mu \sin \theta \sin \zeta \\
\dot{z} & =\dot{z}^{\prime}+\mu \cos \theta
\end{aligned}
$$

We define the characteristic thermal velocity $v_{0}$ as

$$
v_{0}=\left(\frac{2 k T}{m}\right)^{\frac{1}{2}}
$$

Substituting into (39), we obtain

$$
\mathrm{d}^{3} \mathrm{~N}=\mathrm{N}_{\mathrm{O}} \mathrm{f}(\dot{\mathrm{r}}, \dot{\zeta}, \dot{\mathrm{z}}) \operatorname{rd} \dot{\mathrm{r}} \mathrm{d} \dot{\zeta} \mathrm{d} \dot{\mathrm{z}}
$$


where

$$
\begin{aligned}
\mathrm{f}(\dot{\mathrm{r}}, \dot{\zeta}, \dot{\mathrm{z}}) & =\left(\pi^{\frac{1}{2} \mathrm{~V}_{\mathrm{o}}}\right)^{-3} \exp \left\{\frac { 1 } { \mathrm { v } _ { 0 } ^ { 2 } } \left[(\dot{\mathrm{r}}-\mu \sin \theta \cos \zeta)^{2}+\right.\right. \\
& \left.\left.+(\mathrm{r} \dot{\zeta}-\mu \sin \theta \sin \zeta)^{2}+(\dot{z}-\mu \cos \theta)^{2}\right]\right\}
\end{aligned}
$$

The differential of the current to probe is given by

$$
d^{5} i=\dot{z e} d^{3} \mathrm{NdA}
$$

where the differential of the area is in the $r-\zeta$ plane. The total current is obtained by integrating (44) over all of velocity space and the $r-\zeta$ plane for $o \leq r \leq a$ except for those particles which are unable to reach the collection due to their angular momenta. These particles are excluded by appropriately adjusting the integration limits.

Since our assumptions have placed no limitation on $\dot{z}$, the limits of its integration are ${ }^{ \pm \infty}$. We perform this integration first by replacing the variable with $\mathrm{x}$ where

$$
\mathrm{x}=\left(\frac{\dot{z}-\mu \cos \theta}{v_{0}}\right)
$$

The integration over $\dot{z}$ is then of the form

$$
I=K \int_{-\infty}^{\infty} x e^{-x^{2}} d x+k \frac{\mu \cos \theta}{v_{0}} \int_{-\infty}^{\infty} e^{-x^{2}} d x
$$

The first term is symmetric about the origin and vanishes while the second term yields

$$
\begin{aligned}
d^{4}{ }_{i} & =n_{i} e \mu \cos \theta\left(\pi^{\frac{1}{2}} v_{0}\right)^{-2} \exp \left\{\frac { 1 } { v _ { 0 } ^ { 2 } } \left[(\dot{r}-\mu \sin \theta \cos \zeta)^{2}+\right.\right. \\
& \left.\left.+\left(\dot{r}_{\zeta}-\mu \sin \theta \sin \zeta\right)^{2}\right]\right\} \mathrm{r}^{2} \mathrm{~d} \dot{r} \mathrm{~d} \dot{\zeta} \mathrm{d} r \mathrm{~d} \zeta
\end{aligned}
$$


We invoke the conservation of energy (Eq. 6) and angular momentum (Eq. 5) to write

$$
\frac{\mathrm{L}^{2}}{\mathrm{r}_{\mathrm{p}}{ }^{2}}-\frac{\mathrm{L}^{2}}{\mathrm{r}^{2}} \leq \dot{\mathrm{r}}^{2}+\frac{2 \phi_{\mathrm{p}}}{\mathrm{m}}
$$

where the unindexed variable refers to a point in the plane $z=0$. The initial radial velocity $(\dot{r})$ is made up of two terms (Eq. 40)

$$
\dot{\mathbf{r}}=\dot{\mathbf{r}}^{\prime}+\mu \sin \theta \cos \zeta
$$

The first $\left(\dot{r}^{?}\right)$ is thermal while the second is that fraction of the "drift" velocity in the radial direction. For a typical satellite and an $\mathrm{O}_{2}{ }^{+}$ion the "drift" velocity corresponds to about 5 volts. Since our interest is restricted to small values of $\theta$, we may therefore neglect $\dot{\mathrm{r}}^{2}$ as small compared to the probe potential term. This assumption makes our angular momenta criteria independent of $\dot{r}$, and we may integrate equation (47) from $-\infty$ to $+\infty$ to obtain

$$
d_{i}^{3}=\frac{n_{i} e \mu \cos \theta}{\pi^{\frac{1}{2}} v_{0}} \exp \left\{\frac{(r \zeta-\mu \sin \theta \sin \zeta)^{2}}{v_{0}{ }^{2}}\right\} r^{2} d \dot{\zeta} d r d \zeta
$$

Rewriting Eq. (48) using Eq. (5) and setting $\dot{\mathrm{r}}=\mathrm{o}$, we obtain

$$
\mathrm{r}^{2} \dot{\zeta}^{2} \leq \frac{2 \phi_{\mathrm{p}}}{\mathrm{m}}\left(\frac{\mathrm{r}_{\mathrm{p}}{ }^{2}}{\mathrm{r}^{2}-\mathrm{r}_{\mathrm{p}}{ }^{2}}\right) \equiv \Sigma^{2}
$$

which places an upper limit on the value of $\dot{\zeta}$ in terms of the radius, hence, the limits of integration of $\dot{\zeta}$ are $\pm \Sigma / r$. We substitute a new variable of integration

$$
\mathrm{y}=\frac{\mathrm{r} \dot{\zeta}-\mu \sin \theta \sin \zeta}{\mathrm{v}_{0}}
$$

which has limits $Y_{1}$ and $Y_{2}$ where 


$$
\begin{array}{ll}
\mathrm{Y}_{2}= \pm \mathrm{b}-\mathrm{c} \sin \zeta & 0 \leq \mathrm{Y}_{2} \leq 1 \\
\mathrm{Y}_{1}= \pm \mathrm{b}+\mathrm{c} \sin \zeta & 0 \leq \mathrm{Y}_{1} \leq 1
\end{array}
$$

and where

$$
\begin{aligned}
b^{2} & =\frac{\phi}{\frac{1}{2} m v_{o}^{2}} \frac{r_{p}{ }^{2}}{r^{2}-r_{p}{ }^{2}} \\
c & =\frac{u}{v_{0}} \sin \theta
\end{aligned}
$$

Substituting into Eq. (49), we obtain

$$
d^{2} i=\frac{1}{2} n_{i} \text { eu } \cos \theta\left\{\frac{2}{\sqrt{\pi}} \int_{0}^{Y_{2}} e^{-y^{2}} d y+\frac{2}{\sqrt{\pi}} \int_{0}^{Y_{1}} e^{-y^{2}} d y\right\} \operatorname{rdrd} \zeta
$$

The terms within the bracket are "error integrals" whose value ${ }^{11}$ as a function of the limit are shown in Fig. 11 along with several approximation functions. The fit for the sine function is relatively good, however, a simpler approximation is the function

$$
\begin{array}{rlrl}
\frac{2}{\sqrt{\pi}} \int_{0}^{Y} e^{-y^{2}} d y & =Y & & Y \leq 1 \\
& =1 & Y & >1
\end{array}
$$

The error integral is the fraction of particles with velocity less than $\mathrm{Y}$ (where $\mathrm{Y}$ is the velocity in units of $\mathrm{v}_{0}$ ). Equation (56) is equivalent to terminating the velocity distribution in the $\mathbf{r} \dot{\zeta}$ direction at $v_{0}$ while maintaining the proper normalization. These approximations are perhaps more graphically displayed if we examine the original distribution functions rather than their integral. The Maxwellian distribution for a single velocity component has the form 
30

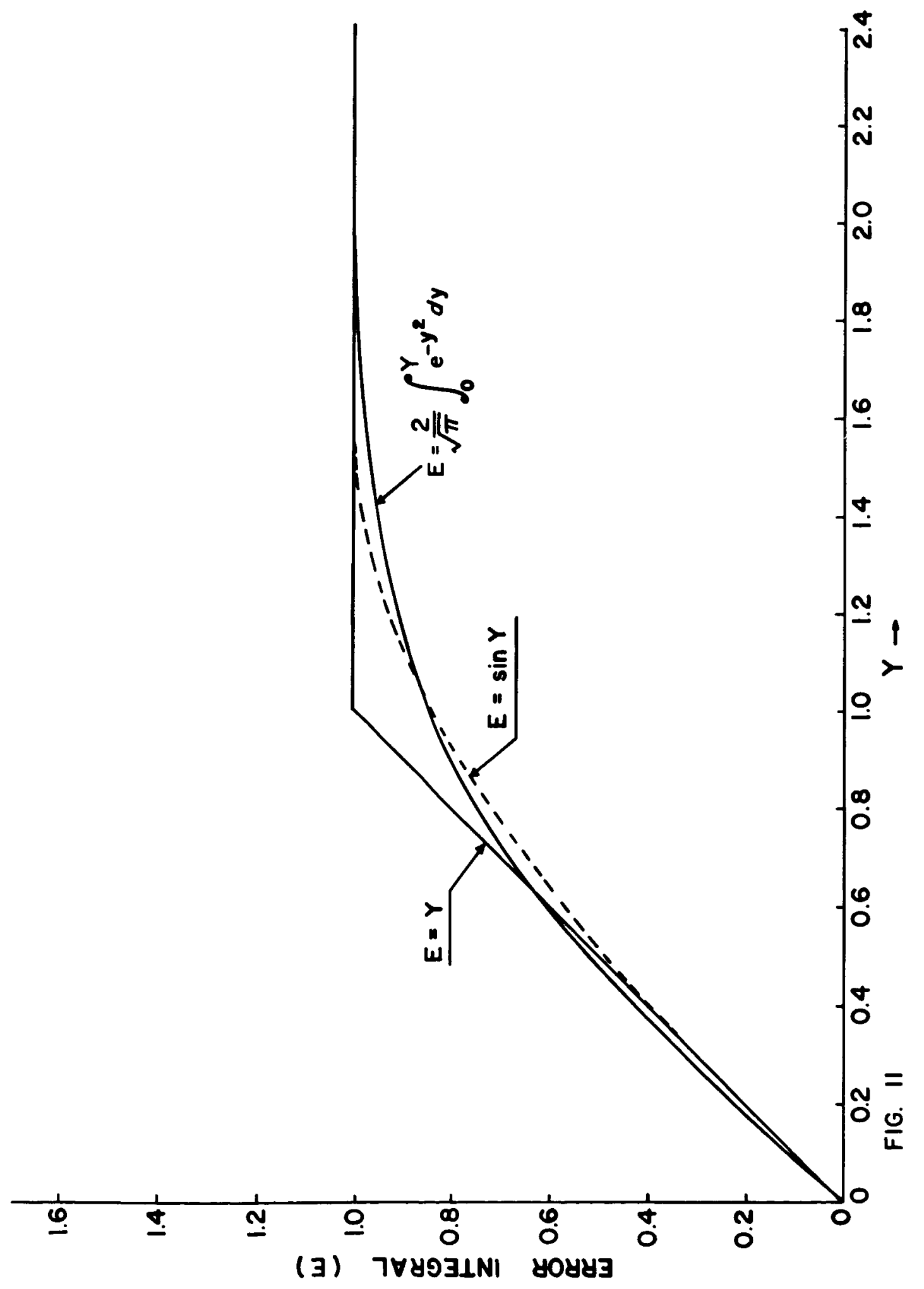




$$
\frac{\mathrm{dN}}{\mathrm{N}}=\frac{1}{\sqrt{\pi}} e^{-\mathrm{y}^{2}} \mathrm{dy}
$$

This is plotted in Fig. 12 along with the generating functions of our other approximations.

We will approach this problem by utilizing the lowest order approximation (equation 56). We make the following substitutions:

$$
\begin{aligned}
& x=r / r_{p} \\
& I=\frac{1}{2 n_{i} \text { eucos } \theta r_{p}^{2}}
\end{aligned}
$$

Equation (58) normalizes the radial coordinate in terms of the probe radius while equation (59) defines a "normalized" current. We may now integrate both sides of equation (55) to obtain:

$$
\begin{aligned}
& I=\int_{-\frac{\pi}{2}}^{\frac{\pi}{2}} \int_{0}^{1} x \mathrm{~d} x \mathrm{~d} \zeta+\int_{-\frac{\pi}{2}}^{-\zeta_{0}} \int_{1}^{\ell} x \mathrm{~d} x \mathrm{~d} \zeta+\int_{-\zeta_{0}}^{\zeta_{0}} \int_{1}^{x_{a}} x \mathrm{~d} x \mathrm{~d} \zeta+\int_{\zeta_{0}}^{\frac{\pi}{2}} \int_{1}^{\ell} x \mathrm{~d} x \mathrm{~d} \zeta \\
& +\int_{-\frac{\pi}{2}}^{\zeta_{3}} \int_{\ell}^{x_{1}}\left(1-Y_{2}{ }^{-}\right) x d x d \zeta+\int_{\zeta_{3}}^{-\zeta_{0}} \int_{\ell}^{x_{a}}\left(1-Y_{2}{ }^{-}\right) x d x d \zeta \\
& -\int_{\zeta_{2}}^{\zeta_{0}} \int_{\mathrm{x}_{1}}^{\mathrm{x}_{\mathrm{a}}}\left(1-\mathrm{Y}_{2}^{+}\right) \mathrm{xdxd} \zeta-\int_{\zeta_{0}}^{\frac{\pi}{2}} \int_{\mathrm{x}_{1}}^{\ell}\left(1-\mathrm{Y}_{2}^{+}\right) \mathrm{xdx} \mathrm{d} \zeta
\end{aligned}
$$

where the superscript $\left(\mathrm{Y}_{2}{ }^{+}, \mathrm{Y}_{2}{ }^{-}\right)$refers to the sign of the leading term. 
32

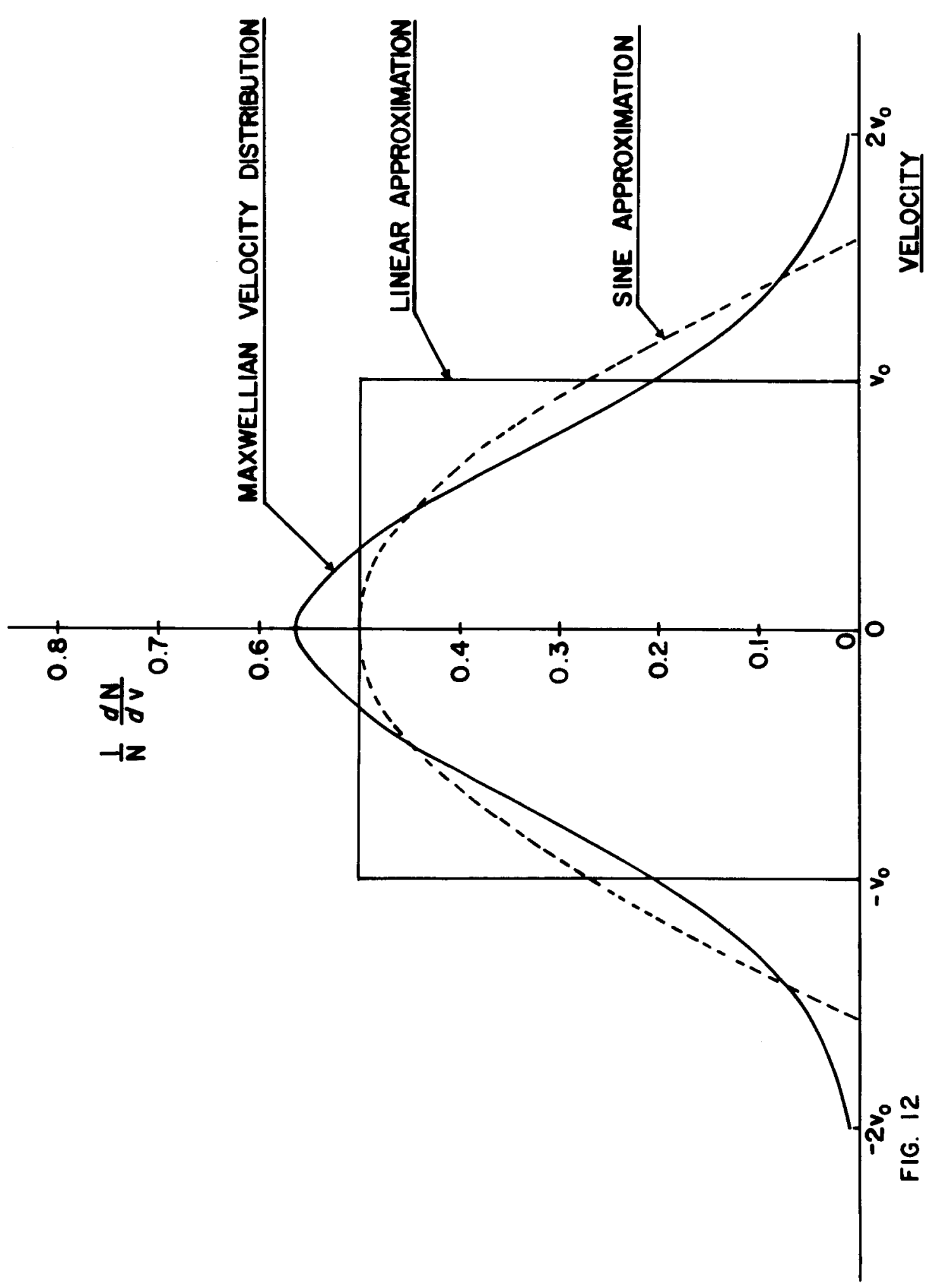


Visualization of these integration limits is aided by figure 13 which represents the $r-\zeta$ plane. The solid curve, bounded by the limits $x_{a}$ and $\ell$ corresponds to the effective collection area for zero velocity in the $\dot{r} \dot{\zeta}$ direction (i.e. no thermal angular momentum). This is the same result we obtained for the cold plasma. The dotted curve corresponds to a particular value of $r \dot{\zeta}$. In the right half plane the angular momenta associated with the drift velocity and the thermal velocity are oppositely directed hence collection is achieved over a larger area. This area is reduced in the left half plane due to the addition of these two terms. Reversing the direction of $\dot{r} \dot{\zeta}$ will simply reflect this pattern about the $\zeta=0$ axis. The area common to both curves represents that portion of the plane for which all particles, regardless of their $r \dot{\zeta}$ velocity, are collected. Collection in the remaining area depends upon this velocity. Note that if the "therma1" angular momentum is greater than that which would allow collection from a radius $x_{a}$ then the limiting angle $\zeta_{2}$ becomes negative.

From symmetry it is clear we only need to integrate over the angular limits from $-\pi / 2$ to $\pi / 2$ and for one direction of $x \dot{\zeta}$ (positive) and then increase our result by a factor of four. The limit $\ell$ is given by the loci of radii for which $y_{2}=0$, that is:

$$
\ell^{2}=1+\frac{\Phi}{c^{2} \sin ^{2} \zeta}
$$

where:

$$
\Phi=\frac{\phi}{\frac{1}{2 m v_{0}^{2}}}
$$




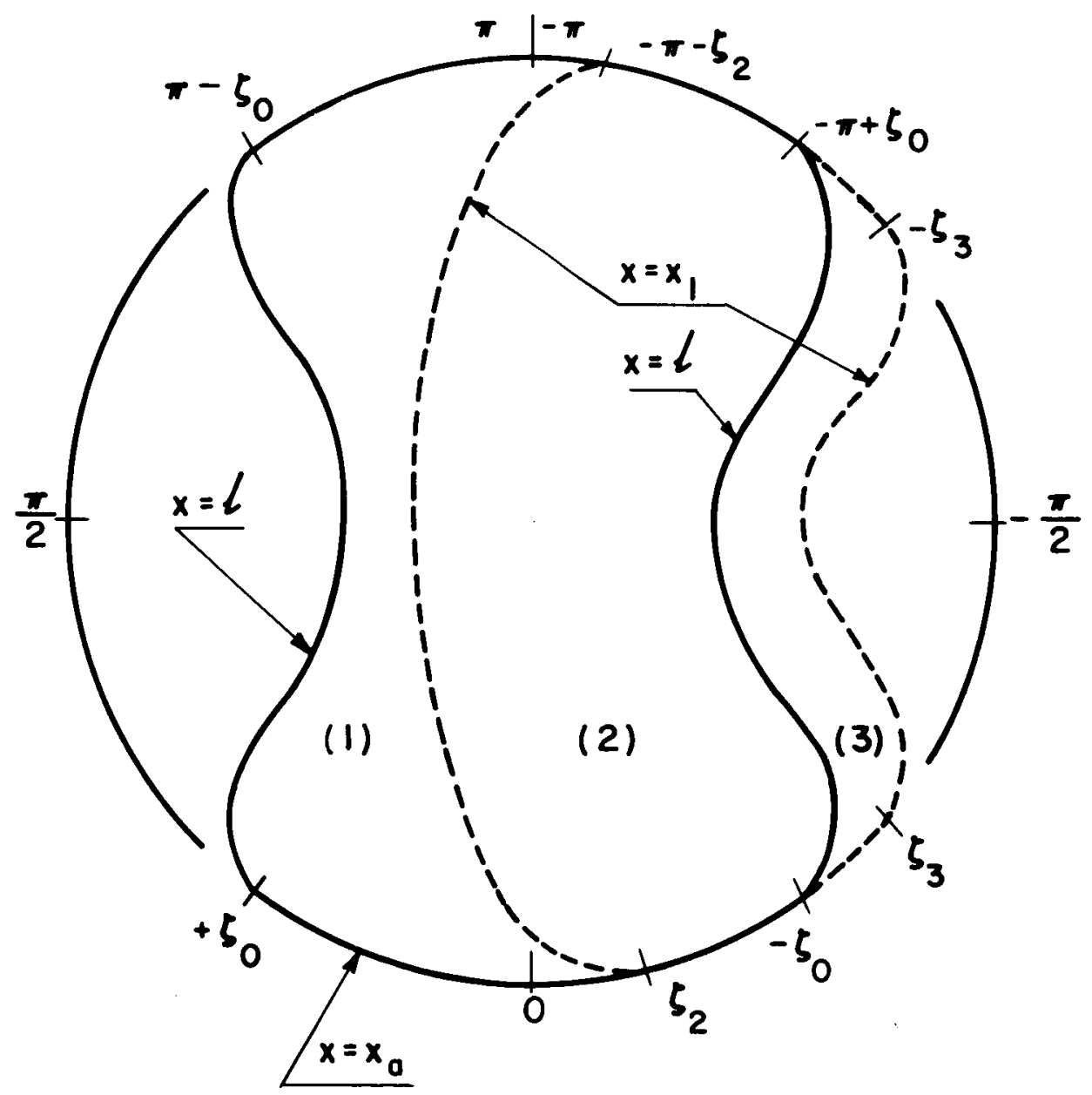

FIG. 13 
The relevant values of $\ell$ are those less than $x_{a}$ hence we define the transistion angle $\zeta_{0}$ by equating $\&$ to $x_{a}$ and obtain:

$$
\sin \zeta_{0}=\frac{b a}{c} \quad 0 \leq\left|\zeta_{0}\right| \leq \frac{\pi}{2}
$$

where $b_{a}$ is the function $b$ evaluated at $x=x_{a}$. The dashed curve is bounded, inside of $x_{a}$ by the curve $x$, for which $Y_{2}=1$, that is:

$$
x_{1}^{2}=1+\frac{\Phi}{(1+c \sin \zeta)^{2}}
$$

The intercept of these curves with the $\mathrm{x}=\mathrm{x}_{\mathrm{a}}$ circle defines two other transistional angles which are given by:

$$
\begin{array}{ll}
\sin \zeta_{2}=-\left(\frac{1-b_{a}}{c}\right) & 0 \leq\left|\zeta_{2}\right| \leq \frac{\pi}{2} \\
\sin \zeta_{3}=-\left(\frac{1+b_{a}}{c}\right) & 0 \leq\left|\zeta_{3}\right| \leq \frac{\pi}{2}
\end{array}
$$

The origin of the terms in equation (60) are now apparent. We will label the areas of figure 13 as 1,2 and 3 . In area one collection depends upon the thermal velocity in the $\mathbf{r} \dot{\zeta}$ direction, hence, collection is proportional to $\mathrm{Y}_{2}^{+}$. In region two all particles are collected while in region three collection is again a function of velocity. In this last case our coefficient is $\left(1-\mathrm{Y}_{2}^{-}\right)$, corresponding to the decrease in particles collected with increasing thermal velocity.

The first term of equation (60) represents collection over the physical tip of the probe; the next three terms are the collection for a caid plasma; the following two terms add in the collection in area three; while the final pair of terms corrects for the effect of velocity in area one. 
Integration of equation (60) yields the result:

$$
\begin{aligned}
I= & \frac{\pi}{2}+\frac{1}{2}\left(x_{a}{ }^{2}-1\right)\left\{\left(\zeta_{2}-\zeta_{3}\right)-2 b_{a}\left(\zeta_{2}+\zeta_{3}\right)-c\left(\cos \zeta_{2}-\cos \zeta_{3}\right)\right. \\
& \left.-b_{a}{ }^{2} F\left(-\frac{\pi}{2}, \zeta_{3}, c\right)-b_{a}{ }^{2} F\left(\zeta_{2}, \frac{\pi}{2}, c\right)\right\}
\end{aligned}
$$

where:

$$
F(\alpha, \beta, c)=\int_{\alpha}^{\beta} \frac{d \zeta}{1+c \sin \zeta}
$$

The form of the " $F$ " function depends upon the absolute value of $c$ :

$$
\begin{aligned}
F(\alpha, \beta, c) & =\left.\frac{2}{\sqrt{1-c^{2}}} \arctan \frac{\tan \frac{\zeta}{2}+c}{\sqrt{1-c^{2}}}\right|_{\alpha} ^{\beta} & |c|<1 \\
& =-\left.c \tan \left(\frac{\pi}{4}-c \frac{\zeta}{2}\right)\right|_{\alpha} ^{\beta} & |c|=1 \\
& =\frac{1}{\sqrt{c^{2}-1}} \log \left\{\left.\frac{\tan \frac{\zeta}{2}+c-\sqrt{c^{2}-1}}{\tan \frac{\zeta}{2}+c+\sqrt{c^{2}-1}}\right|_{\alpha} ^{\beta}\right\} & |c|>1
\end{aligned}
$$

The angle $\zeta_{3}$ is limited to the value $\frac{\pi}{2}$ for $c<1$ hence the last term of equation (67) only involves the general form (69c) except as a limiting case. Note that for $c<1$ :

$$
\mathrm{F}\left(-\frac{\pi}{2},-\zeta_{3}, \mathrm{c}\right) \rightarrow \mathrm{F}\left(-\frac{\pi}{2},-\frac{\pi}{2}, \mathrm{c}\right) \equiv 0
$$

The F function is sometimes troublesome to evaluate for values of c very close to unity. This point is treated in Appendix I. Our expression (equation 67) must properly reduce under various limiting conditions. One of the more important of the limiting conditions 
is that of a cold plasma, i.e. $v_{0} \rightarrow o$. In this limit both $b$ and $c$ diverge, however, their ratio does not:

$$
\left(\frac{b}{c}\right)^{2}=\frac{\phi}{\frac{1}{2 m u^{2} \sin ^{2} \theta}} \frac{r_{p}^{2}}{r^{2}-r_{p}^{2}}
$$

As $v_{0} \rightarrow 0, c \rightarrow \infty,-\zeta_{3} \rightarrow \zeta_{0}$, and $\zeta_{2} \rightarrow \zeta_{0}$. Let us consider the bracketed term of equation (67). In this limit:

$$
\left(\zeta_{2}-\zeta_{3}\right) \rightarrow 2 \zeta_{0}
$$

As $v_{0}$ becomes smal1, we may write:

$$
\begin{aligned}
\zeta_{2} & =\zeta_{0}-\alpha \\
-\zeta_{3} & =\zeta_{0}+\alpha
\end{aligned}
$$

where $\alpha$ may be obtained by expanding the angles as arc sines and then making a binomial expansion of the individual terms:

$$
\begin{aligned}
\zeta_{2}= & \left(\frac{b_{a}}{c}-\frac{1}{c}\right)+\frac{1}{6}\left(\frac{b_{a}}{c}-\frac{1}{c}\right)^{3}+\frac{3}{40}\left(\frac{b_{a}}{c}-\frac{1}{c}\right)^{5}+\ldots \\
= & \left(\frac{b_{a}}{c}\right)+\frac{1}{6}\left(\frac{b_{a}}{c}\right)^{3}+\frac{3}{40}\left(\frac{b_{a}}{c}\right)^{5}+\ldots \\
& -\frac{1}{c}-\frac{1}{2 c}\left(\frac{b_{a}}{c}\right)^{2}-\frac{3}{8 c}\left(\frac{b_{a}}{c}\right)^{4}-\ldots \\
& +\frac{1}{2 c^{2}}\left(\frac{b_{a}}{c}\right)+\frac{3}{4 c^{2}}\left(\frac{b_{a}}{c}\right)^{3}+\ldots
\end{aligned}
$$

Therefore, as c becomes large and we neglect terms of the order of $\frac{1}{c^{2}}$ and higher, we obtain:

$$
\alpha=\frac{1}{c}\left\{1+\frac{1}{2}\left(\frac{b a}{c}\right)^{2}+\frac{3}{8}\left(\frac{b_{a}}{c}\right)^{4}+\ldots .\right\}
$$


Equation (72b) may be verified by a similar expansion for $-\zeta_{3}$. We now note

$$
\mathrm{T}_{2}=-2 \mathrm{~b}_{\mathrm{a}}\left(\zeta_{2}+\zeta_{3}\right) \rightarrow 4 c \alpha \sin \zeta_{0}
$$

The cosine term may be expanded using equation 72 to obtain

$$
\mathrm{T}_{3}=-c\left(\cos \zeta_{2}-\cos \zeta_{3}\right) \rightarrow-2 c \alpha \sin \zeta_{0}
$$

We note, however, that $\mathrm{T}_{2} \neq-\mathrm{T}_{3}$ as we would expect. We are, therefore, left with a residue at the limit since:

$$
T_{2}+T_{3}=2 c a \sin \zeta_{0} \rightarrow 2 \sin \zeta_{0}\left\{1+\frac{1}{2}\left(\frac{b_{a}}{c}\right)^{2}+\frac{3}{8}\left(\frac{b a}{c}\right)^{4}+\ldots\right\}
$$

This result is, of course, invalid and results from taking the limit of $c \rightarrow \infty$ which violates an earlier assumption. In order to clarify this point consider the form of $\mathrm{Y}_{2}{ }^{+}$:

$$
\mathrm{Y}_{2}+=\mathrm{b}-\mathrm{c} \sin \zeta=\mathrm{c}\left(\frac{\mathrm{b}}{\mathrm{b}_{\mathrm{a}}} \sin \zeta_{0}-\sin \zeta\right) \quad 0 \leq \mathrm{Y}_{2}+\leq 1
$$

since the terms inside the parenthesis are finite, $\mathrm{Y}_{2}{ }^{+}$will diverge as c does, hence, the above residue. Clearly, however, both $T_{2}$ and $\mathrm{T}_{3}$ vanish as the thermal velocity vanishes.

The remaining terms may be most easily treated if we note that we may rewrite the $F$ function in the form:

$$
F\left(-\frac{\pi}{2}, \zeta_{3}, c\right)=F\left(-\zeta_{3}, \frac{\pi}{2},-c\right)
$$

If we multiply the numerator and denominator of $F(\alpha, \beta, c)$ and $F(\alpha, \beta,-c$,$) by (1+c \sin \zeta)$ and $(1-c \sin \alpha)$ inside the integral and then sum the resulting terms, we obtain: 


$$
F(\alpha, \beta, c)+F(\alpha, \beta,-c)=2 \int_{\alpha}^{\beta} \frac{d \zeta}{1-c^{2} \sin ^{2} \zeta}
$$

As c becomes large this approaches the limit:

$$
F(\alpha, \beta, c)+\left.F(\alpha, \beta,-c) \underset{c}{\rightarrow} \rightarrow \infty \frac{2}{c^{2}} \cot \zeta\right|_{\alpha} ^{\beta}
$$

We may, therfore, write:

$$
T_{4}=-b_{a}^{2} F\left(-\frac{\pi}{2}, \zeta_{3}, c\right)-b_{a}^{2} F\left(\zeta_{2}, \frac{\pi}{2}, c\right)
$$

We, therefore, obtain for our limiting form:

$$
\begin{aligned}
& I=\frac{\pi}{2}+\frac{1}{2}\left(x_{a}^{2}-1\right)\left(2 \zeta_{0}+\sin 2 \zeta_{0}\right) \\
& i=n_{i} \operatorname{eucos} \theta\left\{\pi r_{p}^{2}+\left(a^{2}-r_{p}{ }^{2}\right)\left(2 \zeta_{0}+\sin 2 \zeta_{0}\right)\right\}
\end{aligned}
$$

This result is essentially the same as equation (18), but the differences in assumptions are clearly evident. In order to make the expressions identical we must employ the following approximations:

$$
\begin{aligned}
& a \ll r_{p} \\
& \phi \ll U \sin ^{2} \theta
\end{aligned}
$$

Another limiting case of interest is that of the high temperature plasma. In this case:

$$
\begin{aligned}
\zeta_{2} & \rightarrow-\pi / 2 \\
\zeta_{3} & \rightarrow-\pi / 2 \\
I & \rightarrow \pi / 2
\end{aligned}
$$


thus, the only current collected is that incident on the tip of the probe, a result which we might anticipate. In general the thermal velocity tends to "smear" the response and a very high ion temperature completely supresses the effect.

The behavior of this function for small values of $\theta$ is of some interest. As $\theta$ becomes small $\zeta_{0}$ reaches the limit $\pi / 2$. (When this occurs, terms such as $c$ sin $\zeta_{0}$ should be replaced by their alternative form i.e. $b_{a}$ in order to avoid the introduction of errors with this fixed limit for $\zeta_{0} \cdot$ ) Before $\zeta_{0}$ reaches this limit the angle $\zeta_{3}$ reaches the limit $-\pi / 2$ and the right-hand curve for $Y_{2}$ in figure 13 collapses. Further, decreases in $\mathrm{c}$ will cause $\zeta_{2}$ to reach a limit of $\pm \pi / 2$. The sign depends upon the realtive value of $b_{a}$ and 1 . At the transistion value particles located at the sheath edge and with the maximum thermal "angular momentum" are at the limit for collection so that all particles, over the entire area are collected. If the thermal energy is greater than this limit (i.e. $b_{a}<1$ ) then some of the particles near the edge of the sheath are lost. For most practical applications, $b_{a} \ll 1$ and the first case is normally relevant only for very low ion temperatures or very large probes. When $b_{a} \geq 1$, the geometric factor $(T=$ bracketed term of equation) reduces to $\pi$ for sma11 $c$; however, if $b_{a}<1$ then we obtain:

$$
T=2 \pi b_{a}-\frac{\pi b_{a}^{2}}{\sqrt{1-c^{2}}}
$$

For small values of $b_{a}$, the current is proportional to $b_{a}$ and independent of $c$ for the range where $\zeta_{2}=-\frac{\pi}{2}$. The effect of $c$ 
for larger values of $b_{a}$ is to reduce $T$ for increasing values of $c$, again, within the same limits. Thus the current at sma11 angles is simply related to the ion thermal energy $\left(\mathrm{b}_{\mathrm{a}}{ }^{2} \propto \frac{1}{\mathrm{kT}}\right)$.

The assumption of $b_{a} \ll 1$ implies that equation (79) will hold for all values of $c$ up to of the order of unity. When $c=1-b_{a}$, the angle $\zeta_{2}$ becomes less than $\pi / 2$ and at $c=1+b_{a} \zeta_{3}$ also departs from its limiting value. It is clear the $\zeta_{2} \approx \zeta_{3}$ for small values of $b_{a}$ so that the behavior of $T$ is dominated by the term:

$$
T=2 b_{a}\left(\zeta_{2}+\zeta_{3}\right)
$$

over the entire range of $c$. Figure 14 compares the result using only this expression for $T$ and the complete form of equation (67). The difference is less than $5 \%$ for $b_{a}=0.1$ except for $c \approx 1-b_{a}$. The difference for $b_{a}=0.05$ is only a few percent. This result is somewhat better than one would expect simply on the basis of the above arguments. The quality of this approximation is aided by the fact that the terms:

$$
\left(\zeta_{2}-\zeta_{3}\right)-c\left(\cos \zeta_{2}-\cos \zeta_{3}\right)
$$

are of the same order of magnitude and of opposite sign over a wide range of values of the argument $c$, hence cancel each other to first order. The last term involving the $\mathrm{F}$ functions is very small due to its coefficient in $b_{a}{ }^{2}$.

We note that the shape of the curve of equation (80) depends only on $c$, that is, for $b_{a}<1$ and $c \geq 1$, we may write:

$$
\begin{aligned}
& \zeta_{2} \approx \beta-\alpha \\
& \zeta_{3} \approx \beta+\alpha
\end{aligned}
$$

$$
\text { where: } \beta=\sin ^{-1}\left(\frac{1}{c}\right)
$$




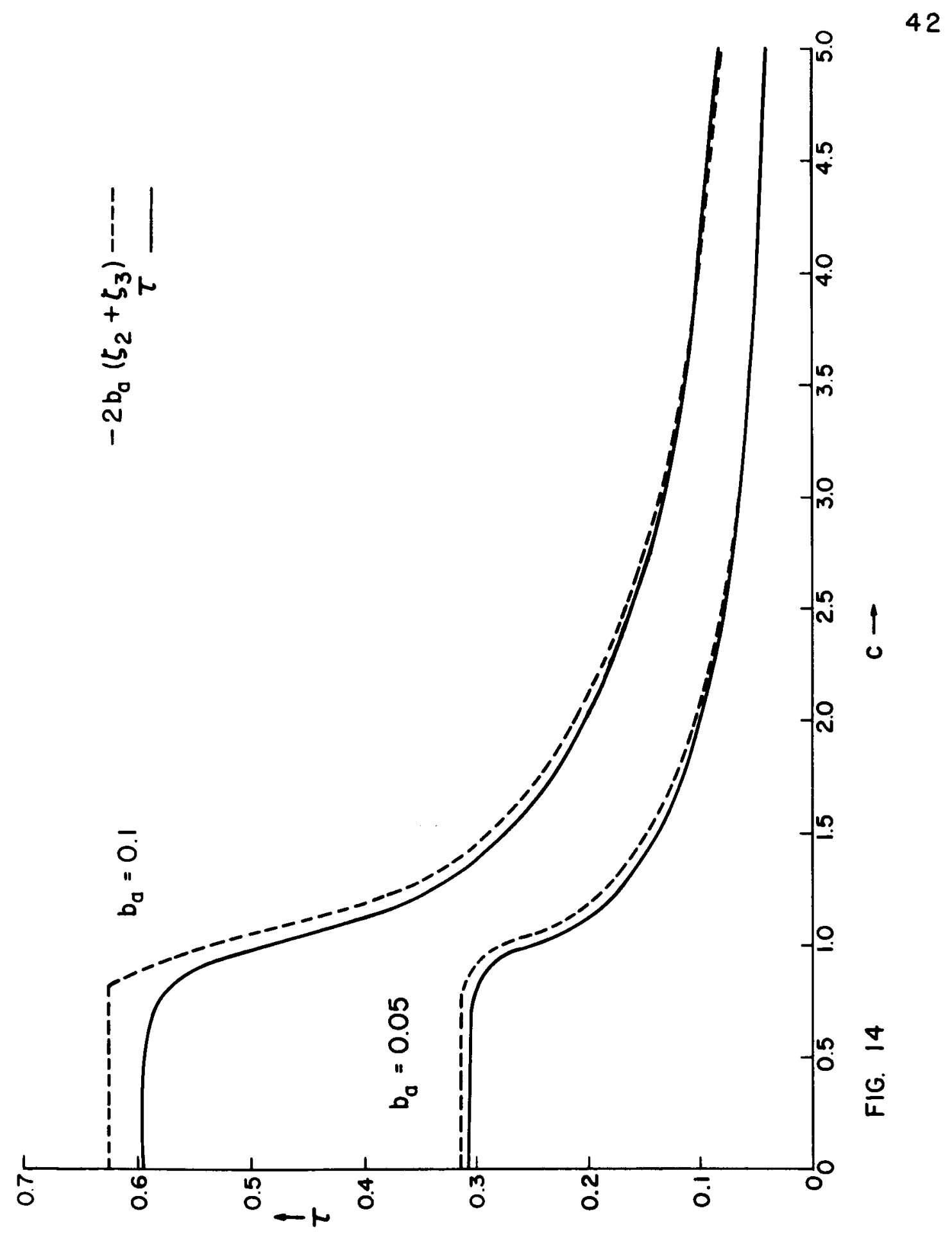


Equation (80) then reduces to:

$$
T \approx 4 b_{a} \sin ^{-1}\left(\frac{1}{c}\right)
$$

Thus the curve falls to one-half of its maximum value at $c=\sqrt{2}$, thus we may relate the ion temperature to the angular half width of $\left(\Theta_{\frac{1}{2}}\right)$ this peak.

$$
\begin{aligned}
\sin \theta_{\frac{1}{2}} & =2 / \mathrm{u}\left(\frac{\mathrm{kT}}{\mathrm{m}}\right)^{\frac{1}{2}} \\
& =\sqrt{2}\left(\frac{\mathrm{v}_{0}}{\mathrm{u}}\right)
\end{aligned}
$$

Thus the curve shape represents a convenient means of determining the ion temperature. Since the width actually depends upon the thermal velocity of the ions their mass also enters with temperature. Another parameter of interest is the probe potential $\left(\phi=e V_{p}\right)$. This is contained within $b_{a}$ however, since the sheath radius also depends upon the potential, we must take this into account. Using equation ( 80$)$, we may write the anomolous current to the probe in the form:

$$
\begin{aligned}
i & =n_{i} \text { eu cos } \theta\left\{\pi r_{p}^{2}+4 r_{p}\left(a^{2}-r_{p}\right)^{\frac{1}{2}} \Phi^{\frac{1}{2}} \sin ^{-1}\left(\frac{1}{c}\right)\right\} \\
& \approx 4 n_{i} \text { eu cos } \theta \text { a } r_{p} \Phi^{\frac{1}{2}} \sin ^{-1}\left(\frac{1}{c}\right)
\end{aligned}
$$

The sheath radius $a$ may be represented in the form ${ }^{10}$ :

$$
a=0.455 \frac{\left(\mathrm{eV}_{\mathrm{p}}\right)^{3 / 4}}{\mathrm{n}_{\mathrm{i}}^{\frac{1}{2}} \mathrm{e}(\mathrm{kT})^{\frac{1}{4}} \ln \left(\frac{\mathrm{a}}{\mathrm{r}_{\mathrm{p}}}\right)}
$$

If we ignore the In term in $a$, then we find that the current is proportional to the power 1.25 of the probe voltage, $\left(\phi=\frac{e V_{p}}{k T}\right)$, 
and inversely to the three-quarter power of ion temperature. Thus the amplitude is independent of the ion mass and dependent on its temperature. Combining equations (83) and (84), we obtain:

$$
i=\frac{1.82 n_{i}^{\frac{1}{2}} \cos \theta r_{p} u\left(e V_{p}\right)^{5 / 4}}{\ln \left(a / r_{p}\right)(k T)^{3 / 4}} \sin ^{-1}\left(\frac{1}{c}\right)
$$




\section{Applications}

This study grew out of some observations by the electrostatic probe on Explorer XVII. Our immediate reaction is to attempt to apply this effect to obtain additional information concerning the ionospheric plasma. The "normal" operation of the electrostatic probe provides data on the electron temperature and charge concentration. It may also be used to supply information on the ion mass distribution ${ }^{13}$. One Important parameter which has been heretofore unavailable is the ion temperature. This end effect shows promise of providing such information.

One of the complications faced in the case of the ionosphere is the mixture of ion masses which often exists. Each constituent will have its own half-width $\left(\Theta_{\frac{1}{2}}\right)$ as given by equation(82b) but fortunately the peak current contribution of each constituent is independent of its mass. Thus the central peak depends only on the ion temperature (assumed to be the same for all ion masses) and the sheath radius. Since we can usually specify the dominate ion mass, we can then obtain the ion temperature and the sheath radius.

This analysis depends upon knowing the current through the end of the probe. Experimentally one obtains the sum of this end current and the more conventional current through the sides of the sheath. If the probe is small $\left(r_{p} \ll a\right)$ and the probe potential large $\left(e v_{p} \gg \mathrm{kT}_{1}\right)$, then this "normal" current is independent of both sheath radius and ion temperature. It takes the form ${ }^{13}$ :

$$
i=2 r_{p} 1_{p} n_{i e}\left(\frac{2}{m}\right)^{\frac{1}{2}}\left(e V_{p}+\frac{1}{2} m u \sin \theta\right)^{\frac{1}{2}}
$$


where $1_{p}$ is the probe length. A third energy term in $k T_{i}$ has been dropped as small compared with $\mathrm{eV}_{\mathrm{p}}$. As $\theta$ approaches zero, this current becomes independent of the "drift" velocity $u$. Even at $\theta=0$, we need to know the distribution of masses in order to properly evaluate this term. According to equation (85) our primary experimentally-controllable parameter which may be used to differenciate between these currents is the probe. The ratio (R) of equations 85 and 86 with $\theta=0$ is:

$$
R=0.64 \frac{m^{\frac{1}{2}} u\left(e v_{p}\right)^{3 / 4}}{n_{i}^{\frac{1}{2}} e I_{p}(k T)^{3 / 4}}\left(\frac{1-\sin ^{2} \theta}{1+\gamma \sin ^{2} \theta}\right)^{\frac{1}{2}} \sin ^{-1}\left(\frac{1}{c}\right)
$$

where: $\quad \gamma=\frac{\mathrm{mu}^{2}}{2 \mathrm{eV}_{\mathrm{p}}}$

The two experimentally controllable parameters which may be used to emphasize the end effect are the probe potential and its length. Both have practical limitations related to the finite length of the probe. You will note that we can define a length $D$, in analogy with the Debye length, utilizing the ion drift energy rather than its thermal energy.

$$
D=\left(\frac{U}{4 \pi n_{1} e^{2}}\right)^{\frac{1}{2}}
$$

For an $0^{+}$ion, a typical satellite velocity of $8 \mathrm{~km} / \mathrm{sec}$ and a density of $10^{4} \mathrm{el} / \mathrm{cc} \mathrm{D}$ has the value $17.2 \mathrm{~cm}$ which is of the same order as a typical probe length L. Using eq. (88), we can rewrite eq. (87) in the form: 


$$
R=2.27\left(\frac{D}{L}\right) \Phi^{3 / 4}\left(\frac{1-\sin ^{2} \theta}{1+\gamma \sin ^{2} \theta}\right)^{\frac{1}{2}} \sin ^{-1}\left(\frac{1}{C}\right)
$$

There exists a value of $\theta$ for which $R \leq 1$. If this angle is greater than the one for which $c=1$, we may reasonably expect to distingquish the end current. These criteria establish a maximum charge concentration $\left(\mathrm{N}_{\mathfrak{1} m}\right)$ in terms of the variables $u, v_{p}, L$ and $T_{i}$.

$$
N_{\text {im }}=\frac{8.2 \mathrm{U}}{\mathrm{e}^{2} \mathrm{~L}^{2}} \quad \Phi^{3 / 2}
$$

Figure 15 is a plot of $N_{i m}$ versus $\Phi$ for various ions, Clearly there exists a maximum density above which this technique can not be used, however, if the probe potential is maintained above 5 or 10 volts this limit is not reached in the terrestrial ionosphere. We conclude that information on ion temperature, sheath dimension and ion mass distribution may be obtained from this technique if the probe is operated at a fixed, large negative potential while being swept through the velocity vector at a rate which is slow relative to the data reading capability.

Chen ${ }^{14}$ has examined some of the Explorer XVII data and finds that, within the limits of accuracy of his data which is only fair, the results agree with the above analysis. The peak halfwidths for $0^{+}$are of the order of $10^{\circ}$. At higher altitudes where $\mathrm{H}^{+}$dominates, the main peak will have a half width of $30^{\circ}$ and, in some cases, wash-out the "normal" peak structure at $90^{\circ}$. This end-effect phenomena may be utilized for many purposes 


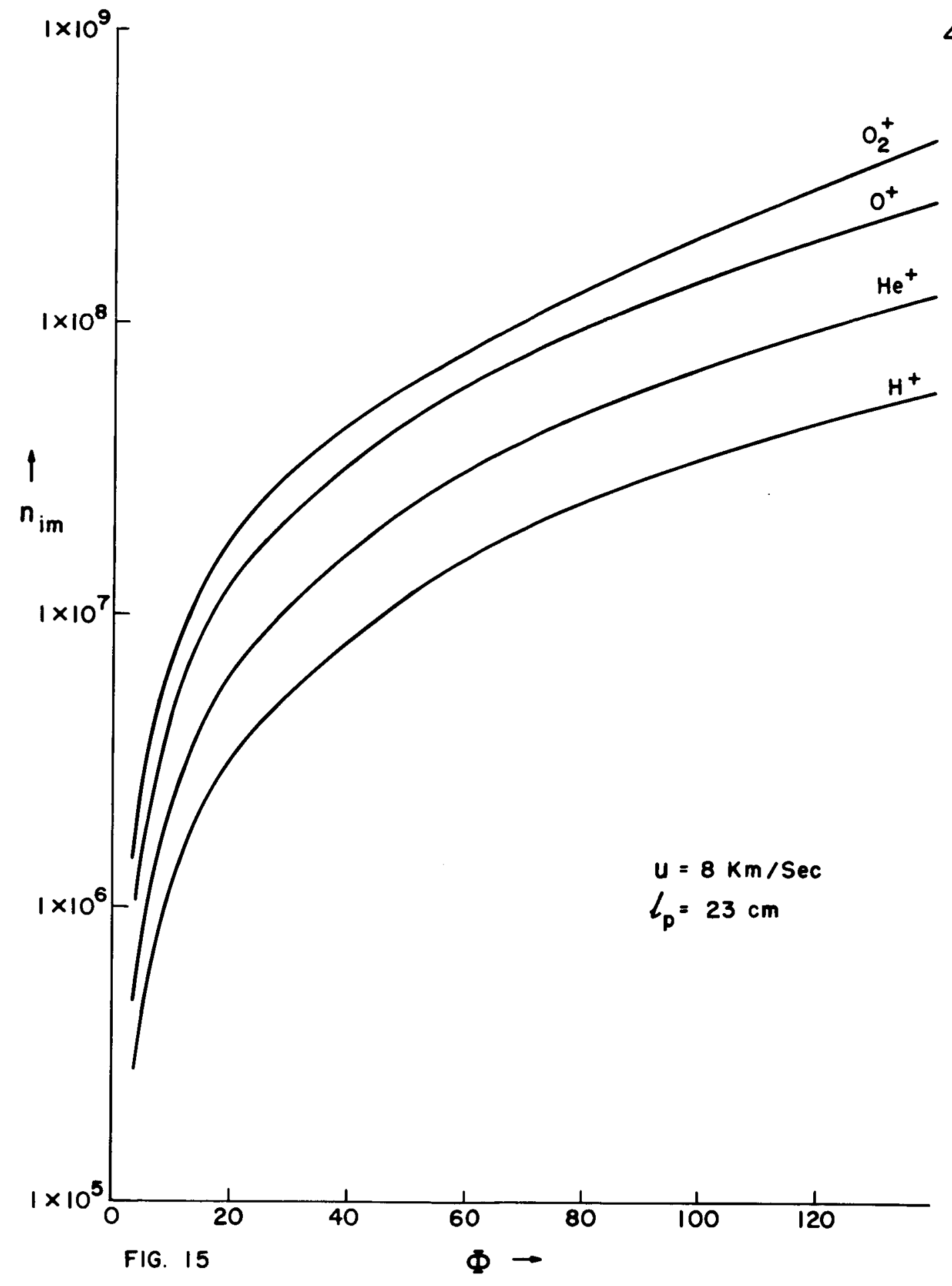


but perhaps one of the more intriguing is its possible use as a means of detecting the velocity vector in a satellite or other high speed vehicle moving within the terrestrial magnetosphere. Several approaches to this problem would seem possible. The first would utilize the major peak structure (i.e. observe $\theta \frac{1}{2}$ for the heaviest significant ion constituent). Unfortunately these half widths are determined by factors not readily controlled by the experimenter. The effects of varying ion temperature and mass distribution would cause this peak width to vary markedly and in some cases to become very wide. The alternative approach would seem to have more promise. This involves observing the change in current while varying the angle $\theta$ over only a few degrees. For this purpose we want the greatest slope in the $i-V$ characteristics at small angles. The fractional change in the current for small values of $c$ may be obtained by differenciating equation (79):

$$
\begin{aligned}
\frac{1}{\mathrm{~T}} \frac{\mathrm{d} \mathrm{T}}{\mathrm{dc}} & =\frac{\mathrm{b}_{\mathrm{a}}\left(\frac{c}{1-c^{2}}\right)}{2\left(1-\mathrm{c}^{2}\right)^{\frac{1}{2}}-b_{a}} \\
& \approx \frac{c}{\left(\frac{2}{\left.b_{a}-1\right)}\right.} \quad c \ll 1
\end{aligned}
$$

This would indicate a maximum slope at $b_{a}=2$, but the equations we have employed lose their validity for this value of the constant. The above analysis applies for $b_{a}$ in the range:

$$
1-|c| \geq b_{a} \geq 0
$$


When $b_{a}$ is larger than this the form of $T$ becomes:

$$
\begin{array}{ll}
\mathrm{T}=\pi \mathrm{b}_{\mathrm{a}} & \mathrm{b}_{\mathrm{a}}=1 \\
\mathrm{~T}=\pi & \mathrm{b}_{\mathrm{a}}>1+|\mathrm{c}|
\end{array}
$$

Thus the slope vanishes for $b_{a}$ outside the range of (92). Thus we conclude that the best resolution is obtained with values of $b_{a}>\frac{1}{2}$. If we vary $c$ up to 0.1 , we will give rise to a change in the current of a few percent which should be quite adequate for these purposes. This amounts to a few degrees in $\theta$, hence, our angular resolution is potentially a small fraction of one degree. There would appear to be a number of promising approaches to detection. The most obvious perhaps involves a conical scan accomplished by mechanically rotating the probe in a conical motion with a half angle of a few degrees. The detected AC modulation of the probe current is phase compared with the mechanical scan from which pitch and yaw error signs are developed. If the device is used simply as a detector rather than a control element, then the output must be normalized with the DC current level.

There are two important objections to this system. Perhaps the most important of the two is the use of a mechanical scan system which presents many problems in a satellite environment, Fortunately there appears to be several alternatives to this arrangement. For Instance, we could use three or four probes evenly spaced on the surface of a small cone. Their mutual interaction would be very small so long as their spacing at the root were large 
compared with their radii. They would share a "common sheath". If $b_{a}$ were small, the current drawn by each probe would be a small fraction of the total and their interaction would clearly be minimal. With $b_{a}$ large, this conclusion is no longer valid and the point requires further investigation. Another possible approach involves the use of a segmented conical probe. 


\section{Limitations}

The foregoing analysis suffers from a number of limitations imposed by the assumptions and approximations utilized in its development. Perhaps the most severe of these is the velocity distribution employed to represent the thermal motions of the particles (equation 56). We have, in effect, eliminated all the high velocity particles and have, thereby, introduced an artificial limft (i.e. $b_{a}>1$ and $\zeta_{2}=\Pi / 2$ for which all particles are collected). We expect that a more exact analysis might soften the current-angle reponse curve somewhat, that is, reduce the central portion of the curve while increasing the wings. The effect should be rather small, but it remains for a more accurate analysis to quantitatively determine its importance.

Several other points not specifically considered in the analysis deserve attention. The integration over the $\dot{z}$ velocity utilized limits of \pm infinity. This is not valid when we consider a practical situation, i.e. a small diameter probe attached to a much larger body. The probe is shielded from particles with velocities in excess of the satellite velocity and similarly directed. Since the mean ion thermal velocity is, throughout most of the ionosphere, small compared to the vehicle velocity this presents no difficulty. In certain regions, generally a high altitude where the ion temperatures are high and the ion masses low, this approximation introduces a significant error. In this case, the limits of integration in one direction should be replaced by $u$. This leads to an additional 
coefficient (K) involving the error function:

$$
K=\frac{1}{2}\left(1+\frac{2}{\sqrt{\pi}} \int_{0}^{x} e^{-y} d y\right)
$$

where $x=\frac{u}{v_{0}}$. When $u \gg v_{0}$ then $k=1$, but if the reverse is true and $u \ll v_{0}$ then $K=\frac{1}{2}$. You will recall from figure 7 that if $x>1.4$ then the error introduced by replacing $k$ with unity is only a few percent.

Another important factor which we have so far neglected is the effect of the finite length of the probe. We have tacitly assumed that all particles entering the sheath will be collected. A finite time is required for the ions to move from their initial radii to the surface of the collector. In general, they have a large velocity parallel to the axis of the probe and might conceivably run out of probe before being collected. Chen ${ }^{14}$ has considered the related problem relevant to the ions incident through the sides of the sheath. He concludes that, at least for the conditions attendant to Explorer XVII, this effect will be negligible for the partcles through the end of the probe. The question depends critically on the potential distribution within the sheath and deserves more attention. The probiem may be avoided of course, by making the probe sufficiently 1ong. This has the disadvantage of increasing the current through the sides of the sheath and increasing the error resulting from estimating this term. 
Appendix I

The evaluation of the function $F(a, b, c)$ where $c \simeq 1.0$ becomes

troublesome because of the coefficient $(|c-1|)^{-\frac{1}{2}}$. This situation is particularly serious when ba $<1$. We may obtain an alternative expression for the function in the following manner:

$$
\Delta=1-\mathrm{c},
$$

where: $\quad|\Delta| \ll 1$

$$
F(a, b, c)=\int_{a}^{b} \frac{d \zeta}{1+c \sin \zeta}=\int_{a}^{b}\left(1-\Delta \frac{\sin \zeta}{1+\sin \zeta}\right)^{-1} \frac{d \zeta}{1+\sin \zeta},
$$

This expression may be approximated by:

$$
\simeq \int_{a}^{b} \frac{d \zeta}{1+\sin \zeta}+\Delta \int_{a}^{b} \frac{\sin \zeta d \zeta}{(1+\sin \zeta)^{2}}
$$

The first integral has the standard solution:

$$
\int_{a}^{b} \frac{d \zeta}{1+\sin \zeta}=-\left.\tan \frac{1}{2}\left(\frac{\pi}{2}-\zeta\right)\right|_{a} ^{b},
$$

We approach the second term by multiplying numerator and demoninator by $(1-\sin \zeta)^{2}$

$$
\begin{aligned}
& \int_{a}^{b} \frac{\sin \zeta d \zeta}{(1+\sin \zeta)^{2}}=\int_{a}^{b} \frac{\sin \zeta\left(1-2 \sin \zeta+\sin ^{2} \zeta\right)}{\cos 4 \zeta} d \zeta \\
& =\int_{a}^{b} \sec ^{2} \zeta \tan \zeta \sec \zeta d \zeta-2 \int_{a}^{b} \tan ^{2} \zeta \sec ^{2} \zeta d+\int_{a}^{b} \tan ^{2} \zeta \tan \zeta d \zeta d \zeta \\
& =\int_{a}^{b} \sec ^{2} \zeta d(\sec \zeta)-2 \int_{a}^{b} \tan ^{2} \zeta d(\tan \zeta)+\int_{a}^{b} \sec ^{2} \zeta d(\sec \zeta)-\int_{a}^{b} d(\sec \zeta)
\end{aligned}
$$


Appendix I continued

$$
=\left.\frac{1}{3}\left\{\sec ^{3} \zeta-2 \tan ^{3} \zeta-3 \sec \zeta\right\}\right|_{a} ^{b} .
$$

This may be rewritten in the form:

$$
\begin{aligned}
2 \sec ^{3} \zeta & -2 \tan ^{3} \zeta-3 \sec \zeta=\sec ^{3} \zeta\left(3 \sin ^{2} \zeta-2 \sin ^{3} \zeta-1\right) \\
& =-2 \sin \zeta \sec ^{3} \zeta\left(\sin ^{2} \zeta-2 \sin \zeta+1-1+\frac{1}{2} \sin \zeta+\frac{1}{2} \sin ^{-1} \zeta\right) \\
& =\frac{-2 \sin \zeta \cos \zeta\left\{(1-\sin \zeta)^{2}+\frac{1}{2} \sin ^{-1} \zeta(1-\sin \zeta)^{2}\right\}}{\left(1-\sin ^{2} \zeta\right)^{2}} \\
& =\frac{\cos \zeta(1+2 \sin \zeta)}{(1+\sin \zeta)^{2}} \\
& =-\frac{\sqrt{\cos \zeta}(1+2 \sin \zeta)}{(\sec \zeta+\tan \zeta)^{2}} \\
& =-\sqrt{\cos \zeta}(1+2 \sin \zeta) \cdot \tan ^{2}\left(\frac{\pi}{4}-\frac{\zeta}{2}\right)
\end{aligned}
$$

Combining terms we obtain:

$$
\begin{aligned}
F(a, b, c) & =-\tan \left(\frac{\pi}{4}-\frac{\zeta}{2}\right)-\left.(1-c) \sqrt{\cos \zeta}(1+2 \sin \zeta) \tan ^{2}\left(\frac{\pi}{2}-\frac{\zeta}{2}\right)\right|_{a} ^{b} \\
c & \simeq 1
\end{aligned}
$$




\section{Acknowledgements}

I am pleased to acknowledge the assistance of L. H. Brace who brought this problem to our attention and has supplied the experimental data; and of F. Huang who has checked the mathematics. A portion of the computer time for this project was supported by the facilities of the Computer Science Center of the University of Maryland.

This work has been supported in part by the National Aeronautics and Space Administration Grant NGR-21-002-057. 


\section{REFERENCES}

1. L. H. Brace, Private Communication.

2. H. M. Mott-Smith and I. Langmuir, Phys. Rev. 28, 727 (1926).

3. L. H. Brace and N. W. Spencer, J. Geophys. Research $\underline{69}, 4686$ (1964).

4. E. J. "Opik, in Interaction of Space Vehicles and an Ionized Atmosphere, edited by S. F. Singer (Pergamon Press, London, 1965).

5. I. B. Berstein and I. N. Rabinowitz, Project Matterhorn Report PM-S-38, (October 1958).

6. L. P. Pitaevskii, AIAA I, 994 (1963).

7. A. H. Davis and I. Harris, in Rarefield Gas Dynamics, edited by L. Talbot (Academic Press, New York, 1961), pp.691.

8. E. H. Walker, in Interactions of Space Vehicles and an Ionized Atmosphere, edited by S. F. Singer (Pergamon Press, London, 1965).

9. E. H. Walker, Private Communication.

10. R. T. Bettinger and E. H. Walker, Phys. of Fluids $\underline{8}, 748$ (1965).

11. E. Johnke and F. Emde, Tables of Functions (Dover Publications, New York, 1945).

12. Standard Mathematical Tables (The Chemical Rubber Co., Cleveland, 1964).

13. 'R. T. Bettinger, Univ. of Maryland, Dept. of Physics and Astronomy Technical Report 484 (August 1965).

14. A. A. Chen, Univ. of Maryland, Dept. of Physics and Astronomy Technical Report 612 (August 1966). 